\title{
ORIGINAL
}

\section{Epidemiology of intra-abdominal infection and sepsis in critically ill patients: "AbSeS", a multinational observational cohort study and ESICM Trials Group Project}

Stijn Blot ${ }^{{ }^{*}}$ (1), Massimo Antonelli ${ }^{2,3}$, Kostoula Arvaniti $^{4}$, Koen Blot ${ }^{1}$, Ben Creagh-Brown ${ }^{5,6}$, Dylan de Lange ${ }^{7}$, Jan De Waele ${ }^{8}$, Mieke Deschepper ${ }^{9}$, Yalim Dikmen $^{10}$, George Dimopoulos ${ }^{11}$, Christian Eckmann ${ }^{12}$, Guy Francois $^{13}$, Massimo Girardis ${ }^{14}$, Despoina Koulenti ${ }^{15,16}$, Sonia Labeau ${ }^{1,17}$, Jeffrey Lipman ${ }^{18,19}$, Fernando Lipovestky ${ }^{20}$, Emilio Maseda ${ }^{21}$, Philippe Montravers ${ }^{22,23}$, Adam Mikstacki ${ }^{24,25}$, José-Artur Paiva ${ }^{26}$, Cecilia Pereyra ${ }^{27}$, Jordi Rello ${ }^{28}$, Jean-Francois Timsit ${ }^{29,30}$, Dirk Vogelaers ${ }^{31}$ and the Abdominal Sepsis Study (AbSeS) group on behalf of the Trials Group of the European Society of Intensive Care Medicine

() 2019 The Author(s)

\begin{abstract}
Purpose: To describe the epidemiology of intra-abdominal infection in an international cohort of ICU patients according to a new system that classifies cases according to setting of infection acquisition (community-acquired, early onset hospital-acquired, and late-onset hospital-acquired), anatomical disruption (absent or present with localized or diffuse peritonitis), and severity of disease expression (infection, sepsis, and septic shock).

Methods: We performed a multicenter $(n=309)$, observational, epidemiological study including adult ICU patients diagnosed with intra-abdominal infection. Risk factors for mortality were assessed by logistic regression analysis.

Results: The cohort included 2621 patients. Setting of infection acquisition was community-acquired in 31.6\%, early onset hospital-acquired in $25 \%$, and late-onset hospital-acquired in $43.4 \%$ of patients. Overall prevalence of antimicrobial resistance was $26.3 \%$ and difficult-to-treat resistant Gram-negative bacteria 4.3\%, with great variation according to geographic region. No difference in prevalence of antimicrobial resistance was observed according to setting of infection acquisition. Overall mortality was $29.1 \%$. Independent risk factors for mortality included late-onset hospitalacquired infection, diffuse peritonitis, sepsis, septic shock, older age, malnutrition, liver failure, congestive heart failure, antimicrobial resistance (either methicillin-resistant Staphylococcus aureus, vancomycin-resistant enterococci, extended-spectrum beta-lactamase-producing Gram-negative bacteria, or carbapenem-resistant Gram-negative bacteria) and source control failure evidenced by either the need for surgical revision or persistent inflammation.
\end{abstract}

\footnotetext{
*Correspondence: stijn.blot@UGent.be

${ }^{1}$ Department of Internal Medicine and Pediatrics, Ghent University, Campus UZ Gent, Corneel Heymanslaan 10,9000 Ghent, Belgium

Full author information is available at the end of the article
}

The members of the Abdominal Sepsis Study (AbSeS) group for the Trials Group of the European Society of Intensive Care Medicine have been given in the Acknowledgements section.

\section{实 Springer}


Conclusion: This multinational, heterogeneous cohort of ICU patients with intra-abdominal infection revealed that setting of infection acquisition, anatomical disruption, and severity of disease expression are disease-specific phenotypic characteristics associated with outcome, irrespective of the type of infection. Antimicrobial resistance is equally common in community-acquired as in hospital-acquired infection.

Keywords: Intra-abdominal infection, Peritonitis, Sepsis, Intensive care, Multidrug resistance, Mortality

\section{Introduction}

Severe intra-abdominal infections are a frequent and important issue in intensive care (ICU). According to international literature, the abdomen often ranks first or second among the sources of infection or sepsis [1-3].

Intra-abdominal infections pose several particular clinical challenges. First, there is a large span of disease severity ranging from uncomplicated cases to fulminant septic shock and multi-organ dysfunction. Second, there is the broad spectrum of pathogens including Gram-positive and Gram-negative aerobic bacteria, anaerobes, and fungi [4]. Third, the contribution of microbiological diagnosis is not straightforward as cultures cannot always readily discriminate true pathogens from harmless micro-organisms [5, 6]. Furthermore, source control encompassing all interventions to eradicate the source of infection, control on-going contamination, and to restore anatomic derangements and physiologic function, is key to clinical management and success, but often difficult to achieve [5, 7, 8]. Finally, there is the wide variety of clinical entities within intraabdominal infections. Besides local abscess formation or solid organ infection (e.g., liver abscesses and infected pancreatic necrosis), a classic approach recognizes three types of peritonitis: i.e., primary peritonitis (peritoneal dialysis-related or spontaneous bacterial peritonitis), secondary peritonitis (following anatomical disruption of the GI tract), or tertiary peritonitis (persistent infection despite adequate source control intervention). In addition, cases of intra-abdominal infection are often classified as uncomplicated or complicated. Complicated describes extension of infection from their source into the peritoneal cavity.

Because of this heterogeneity, the intra-abdominal infections are difficult to study [9]. To bring more clarity in the terminology, an alternative classification for intra-abdominal infections has been proposed [10]. This system classifies intra-abdominal infections according to their setting of acquisition (community-acquired, healthcare-associated or early onset hospital-acquired, or late-onset hospital-acquired), presence of anatomical disruption (either absent or present resulting in localized or diffuse peritonitis), and severity of disease expression (infection, sepsis, or septic shock). This classification

\section{Key message}

A multinational epidemiological study on intra-abdominal infection in ICU patients revealed that setting of infection acquisition, anatomical barrier disruption, and severity of disease expression are disease-specific phenotypic characteristics associated with mortality.

Antibiotic resistance appeared equally in community-acquired as in hospital-acquired infection.

defines different phenotypes of the same disease (e.g., diverticulitis) by covering aspects of (i) the extent of intra-abdominal contamination reflecting the complexity of source control, (ii) level of associated organ failure indicating sense of urgency and prognosis, and (iii) likelihood of antimicrobial resistant micro-organisms or otherwise important pathogens which may require broader antimicrobial coverage (enterococci, Candida spp.).

The objective of the study was to describe the epidemiology of intra-abdominal infection in an international cohort of ICU patients and to validate the predictive value for mortality of an alternative classification system.

\section{Methods}

A complete version of the Methods is in Supplement-1. $\mathrm{AbSeS}$ was an international, multicenter, prospective observational cohort study conducted between January and December 2016. Consecutive, adult ICU patients diagnosed with intra-abdominal infection, either as primary diagnosis leading to ICU admission or as a complication occurring during the ICU course, were eligible for inclusion. Overall, approval by established national, regional, or local institutional review boards was expedited and granted. The study is registered at ClinicalTrials.gov (number NCT03270345).

\section{Data recorded and definitions}

We obtained data describing the hospital and intensivecare facility through a center report form. Anonymous patient data were collected through the case report form. Examples of the center and case report forms are in Supplement-2. Type of intra-abdominal infection was defined according to the International Sepsis Forum Consensus Conference Definitions [11]. Intra-abdominal infections were classified according to setting of infection 
acquisition, anatomical barrier disruption, and severity of disease expression [10]. Setting is community-acquired, healthcare-associated and/or early onset hospitalacquired ( $\leq 7$ days of hospital admission), or late-onset hospital-acquired ( $>7$ days of hospital admission [12]). Healthcare-associated onset is defined by at least one of the following risk factors for multidrug-resistant pathogens: nursing home resident, out-of-hospital parenteral nutrition or vascular access, chronic dialysis, recent hospital admission ( $<6$ months), or recent antimicrobial therapy ( $<6$ months). For convenience sake, 'healthcareassociated and/or early-onset hospital-acquired' cases are designated 'early-onset hospital-acquired'. Intra-abdominal infections were classified as either without anatomical disruption, or with anatomical disruption resulting in localized or diffuse peritonitis (i.e., contamination spread to entire abdominal cavity). Severity of disease expression is defined as either infection, sepsis, or septic shock [13]. Microbiological assessment was left at the discretion of the physician. Eligible cultures included intra-operative cultures, trans-abdominal fine-needle aspiration, blood cultures presumably related to the intra-abdominal infection, and cultures from abdominal drains sampled $\leq 24 \mathrm{~h}$ post-surgery. Thresholds for resistance were those as reported by The European Committee on Antimicrobial Susceptibility Testing (EUCAST) [14]. Antimicrobial resistance was defined as methicillin resistance for Staphylococcus aureus, vancomycin resistance for enterococci, and for Gram-negative bacteria either production of extended-spectrum beta-lactamase (ESBL), carbapenem resistance, or fluoroquinolone resistance (resistance against ciprofloxacin, levofloxacin, or moxifloxacin). To assess relationships between resistance and mortality, we also used the definition of "difficult-totreat" resistance for Gram-negative bacteria. This combines resistance to all tested carbapenem, beta-lactam, and fluoroquinolone agents, and is associated with worse clinical outcomes in bloodstream infection $[15,16]$. We deviated from this definition, however, using ESBL production as a proxy for resistance against penicillins, cephalosporins, and monobactams. For reporting microbiological results, the number of patients with cultures sampled is used as denominator. Data on anti-infective management included antimicrobial therapy and source control. Antimicrobial coverage of empiric therapy was evaluated for basic coverage (i.e., coverage of Grampositive, Gram-negative, and anaerobic bacteria), and the association of an antimicrobial agent or initial choice with potential clinical activity against Pseudomonas aeruginosa, methicillin-resistant S. aureus (MRSA), enterococci, vancomycin-resistant enterococci (VRE), and Candida. In this regard, coverage of enterococci targets
Enterococcus faecalis [6]. Outcome data included source control assessment 7 days post-diagnosis or earlier if the patient died within that time window. Source control was judged as either successful or having failed. Failure represented either persistent inflammation (clinical evidence of a remaining source of infection) or the necessity of re-intervention following the initial approach (conservative management or source control intervention). Main outcome is ICU mortality with a minimum of 28 days of observation.

\section{Data management and statistical analyses}

Simple descriptive statistics were used to characterize the study population; continuous data were summarized by median and interquartile range, categorical data as $n$ (\%). Logistic regression analysis was used to assess relationships with mortality. Details on the regression models are in Supplement-1. It can be considered inappropriate to include 'source control achievement at day 7' in the model as this covariate is instrumental to the biological pathway between infection onset and mortality. Therefore, we report a logistic regression model with and without source control achievement.

\section{Results}

During the study period, 2850 patients were included; 229 were excluded, because essential data were missing. As such, 2621 patients from 309 ICUs from 42 countries were entered for analysis. Most patients were included in various European regions $(n=1830 ; 69.8 \%)$, followed by Middle \& South America $(n=366 ; 14.0 \%)$, North Africa \& Middle-East $(n=214 ; 8.8 \%)$, AsiaPacific $(n=174 ; 6.6 \%)$, North America $(n=29 ; 1.1 \%)$, and Sub-Saharan Africa ( $n=8 ; 0.3 \%$ ) (Supplement-3).

Characteristics of the study cohort according to setting of infection acquisition are reported in Table 1. Setting of infection acquisition was communityacquired in 828 patients (31.6\%), early onset hospital-acquired in 656 patients (25.0\%), and late-onset hospital-acquired in 1137 patients (43.4\%). Underlying conditions were more frequently observed in cases with healthcare-associated or hospital-acquired infection. Cases with hospital-acquired infection had higher SOFA scores and more often septic shock.

The vast majority of cases involved secondary peritonitis $(68.4 \%)$, followed by biliary tract infection (12.2\%), intra-abdominal abscess (6.9\%), and pancreatic infection (6.3\%). Primary peritonitis, toxic megacolon, peritoneal dialysis-related peritonitis, and typhlitis were less frequent $(<4 \%)$. Details on the distribution according to setting of infection acquisition are reported in Table 2. 


\section{Microbiology}

Microbiological samples were obtained in 1982 patients (75.6\%). In $80.4 \%$ of these patients, at least one culture was found positive $(n=1594)$. Figure 1 reports the type of samples obtained with their respective proportion of culture positivity. Gram-negative bacteria were most frequently isolated (58.6\%) with Enterobacterales as predominant family (51.7\%) and Escherichia coli as most common pathogen (36.8\%). Gram-positive aerobic bacteria were isolated in $39.4 \%$ of patients with enterococci as most prevalent species (25.9\%). Furthermore, anaerobic bacteria and fungi were isolated in $11.7 \%$ and $13.0 \%$ of patients, respectively. Detailed results on isolated micro-organisms are reported in Table 3. Multidrug-resistant micro-organisms were isolated from 522 patients (26.3\%). Antimicrobial resistance rates were not different among community-acquired (26.5\%), early onset hospital-acquired (29.0\%), and lateonset hospital-acquired infection (24.6\%) $(p=0.215)$. There was also no difference in antimicrobial resistance among patients with infection (27.6\%), sepsis (26.9\%), and septic shock $(25.0 \%)(p=0.449)$. Antimicrobial resistance is mainly a matter of Gram-negatives, but variations according to geographic region are substantial (Table 4). Regions of particular concern include Eastern- and South-East Europe, North Africa and the Middle-East, and Latin America as $>35 \%$ of patients are infected by at least one antimicrobial resistant microorganism. Antimicrobial resistance rates according to setting of infection acquisition and region are reported in Supplement-5.

\section{Antimicrobial therapy}

Data on the first-line empiric antimicrobial therapy was available from 2427 patients (92.6\%). A basic schedule covering aerobic Gram-positive, Gram-negative, and anaerobic bacteria was prescribed in 2291 patients (94.4\%). An anti-pseudomonal agent was prescribed in 1978 patients (81.8\%). Empiric coverage of MRSA and VRE was added in, respectively, 647 patients $(26.7 \%)$ and 140 patients $(5.8 \%)$. An antifungal agent was associated in 436 patients (18\%). In 365 patients, two agents with anti-anaerobic activity were prescribed (15\%). Double anti-anaerobic coverage was more frequently prescribed in hospital-acquired cases (18.2\%) compared with community-acquired cases (14.2\%). No other differences in antimicrobial coverage according to setting of infection acquisition were observed (Supplement-6).

\section{Source control}

Data on the initial approach to control the infection are reported in 2438 patients. A source control intervention was carried out in 2334 patients (95.7\%), and included drainage (94.0\%), decompressive surgery (7.9\%), and restoration of anatomy and function (28.2\%). Among patients undergoing source control, persistent inflammation at day 7 was reported in 692 patients (29.6\%). An additional intervention was deemed necessary in 382 patients (16.4\%). Among patients with an initial conservative approach $(n=104), 30$ patients experienced persistent inflammation (28.8\%), and a source control intervention was performed in 5 patients $(4.8 \%)$. More details on source control interventions and evaluations are summarized in Fig. 2.

\section{Mortality}

Overall mortality was $29.1 \%$ (752/2588). Univariate relationships with mortality are reported in Supplement-7. Mortality stepwise increased with ascending SOFA scores (Supplement-8). Achievement of source control at day 7 was associated with lower mortality (248/1438, 17.2\%) compared with cases with persistent inflammation $(367 / 761,51.8 \%)$ and those requiring surgical revision $(110 / 389,28.3 \%)(p<0.001)$. We reported mortality according to setting of infection acquisition, anatomical disruption, and severity of disease expression. Mortality was $23.7 \%$ in community-acquired cases, $27.3 \%$ in early onset hospital-acquired cases, and $33.9 \%$ in late-onset hospital-acquired cases $(p<0.001)$. Regarding anatomical disruption, no difference in mortality was observed between patients without anatomical disruption and those with localized peritonitis (respectively, 25.0\% and 24.2\%, $p=0.135$ ). Mortality in patients with diffuse peritonitis $(36.0 \%)$ was higher compared with the former categories $(p<0.001)$. Finally, mortality stepwise increased with greater severity of disease expression: $12.8 \%$ in infected patients without sepsis, $24.5 \%$ in septic patients, and $40.3 \%$ in patients with septic shock $(p<0.001)$. Table 5 reports mortality rates for all different phenotypes of intra-abdominal infection according to setting of infection acquisition, anatomical disruption, and severity of disease expression. The grid describes a stepwise increase in mortality along with combinations including septic shock, diffuse peritonitis, and late-onset hospital-acquired infection.

Logistic regression analysis identified late-onset hospital-acquired infection, diffuse peritonitis, sepsis and septic shock, older age, malnutrition, diabetes mellitus, liver failure, and congestive heart failure as independent risk factors for death (Table 6). The association of an antiMRSA agent in the empiric antimicrobial scheme was associated with decreased risk of death. Antimicrobial resistance defined as MRSA, VRE, or difficult-to-treat resistant Gram-negatives did not reached the final models. However, when antimicrobial resistance in Gramnegative bacteria was defined as either ESBL production 
Table 1 Patient characteristics of intensive-care unit patients with intra-abdominal infection/sepsis according to setting of infection acquisition

\begin{tabular}{|c|c|c|c|c|c|}
\hline Characteristic & Total cohort $(n=2621)$ & Community-acquired ( $n=828$ ) & $\begin{array}{l}\text { Early onset hospital- } \\
\text { acquired }(n=656)\end{array}$ & $\begin{array}{l}\text { Late-onset hospital- } \\
\text { acquired ( } n=1137 \text { ) }\end{array}$ & $p^{*}$ \\
\hline \multicolumn{6}{|l|}{ Demographics } \\
\hline Age, years & $66(54-75)$ & $67(52-77)$ & $66(54-77)$ & $66(55-74)$ & 0.213 \\
\hline Sex, male & 1488/2615 (56.9) & $452(54.6)$ & $364(55.5)$ & $672(59.1)$ & 0.133 \\
\hline Type of ICU admission & $2592^{* *}$ & $799^{* *}$ & 656 & 1137 & \\
\hline Medical & $472(18.2)$ & $109(13.7)$ & $131(20.0)$ & $232(20.4)$ & $<0.001$ \\
\hline Surgical, non-emergency & $233(9.0)$ & $19(2.4)$ & $39(5.9)$ & $175(15.4)$ & $<0.001$ \\
\hline Surgical, emergency & $1847(71.3)$ & $660(82.6)$ & $478(72.9)$ & $709(62.4)$ & $<0.001$ \\
\hline Trauma & $40(1.5)$ & $11(1.4)$ & $8(1.2)$ & $21(1.8)$ & 0.496 \\
\hline ICU stay, days & $9(4-18)$ & $9(4-18)$ & $9(4-17)$ & $10(5-19)$ & 0.183 \\
\hline \multicolumn{6}{|l|}{ Underlying conditions*** } \\
\hline Chronic pulmonary disease & $342(13.0)$ & $96(11.6)$ & $90(13.7)$ & $156(13.7)$ & 0.324 \\
\hline AIDS & $14(0.5)$ & $6(0.7)$ & $3(0.5)$ & $5(0.4)$ & 0.661 \\
\hline Malignancy & $699(26.7)$ & $116(14.0)$ & $170(25.9)$ & $413(36.3)$ & $<0.001$ \\
\hline Neurologic disease & $165(6.3)$ & $42(5.1)$ & $60(9.1)$ & $75(6.6)$ & 0.008 \\
\hline Peptic ulcer disease & $176(6.7)$ & $57(6.9)$ & $52(7.9)$ & $67(5.9)$ & 0.246 \\
\hline Liver disease & $127(4.8)$ & $24(1.5)$ & $44(6.7)$ & $59(5.2)$ & 0.002 \\
\hline Chronic renal failure & $282(10.8)$ & $57(6.9)$ & $100(15.2)$ & $125(11.0)$ & $<0.001$ \\
\hline Myocardial infarction & $188(7.2)$ & $48(5.8)$ & $57(8.7)$ & $83(7.3)$ & 0.098 \\
\hline $\begin{array}{l}\text { Chronic heart failure (NY Heart } \\
\text { Association class IV) }\end{array}$ & $184(7.0)$ & $36(4.3)$ & $64(9.8)$ & $84(7.4)$ & $<0.001$ \\
\hline Peripheral vascular disease & $169(6.4)$ & $34(4.1)$ & $48(7.3)$ & $87(7.7)$ & 0.004 \\
\hline Diabetes mellitus & $488(18.6)$ & $116(14.0)$ & $141(21.5)$ & $231(20.3)$ & $<0.001$ \\
\hline Immunosuppression & $253(9.7)$ & $47(5.7)$ & $83(12.7)$ & $123(10.8)$ & $<0.001$ \\
\hline Lifestyle risk factors & $1363(52.0)$ & $413(49.9)$ & $355(54.1)$ & $595(52.3)$ & 0.257 \\
\hline Malnutrition (body mass index $<20$ ) & $177(6.8)$ & $46(5.6)$ & $53(8.1)$ & $78(6.9)$ & 0.154 \\
\hline Obesity (body mass index $\geq 30$ ) & $735(28.0)$ & $236(28.5)$ & $197(30.0)$ & $302(26.6)$ & 0.271 \\
\hline Tobacco use (> 20 pack years) & $446(17.0)$ & $127(7.1)$ & $106(16.2)$ & $213(18.7)$ & 0.113 \\
\hline Alcohol abuse (> $10 \mathrm{~g}$ alcohol/day) & $196(7.5)$ & $59(7.1)$ & $49(7.5)$ & $88(7.7)$ & 0.261 \\
\hline IV drug abuse & $17(0.6)$ & $8(1.0)$ & $3(0.5)$ & $6(0.5)$ & - \\
\hline \multicolumn{6}{|l|}{ Severity of acute illness } \\
\hline $\begin{array}{l}\text { SAPS II score at time of ICU admis- } \\
\text { sion }\end{array}$ & $49(39-60)$ & $48(38-59)$ & $49(39-61)$ & $49(38-60)$ & 0.183 \\
\hline SOFA score at diagnosis & $6(3-10)$ & $5(3-9)$ & $7(3-10)$ & $6(3-10)$ & $<0.001$ \\
\hline \multicolumn{6}{|l|}{ Severity of disease expression } \\
\hline Infection without sepsis & $164(6.3)$ & $51(6.2)$ & $42(6.4)$ & $71(6.2)$ & 0.981 \\
\hline Sepsis & $1590(60.7)$ & $528(63.8)$ & $399(60.8)$ & $663(58.3)$ & 0.050 \\
\hline Septic shock & $867(33.1)$ & $249(30.1)$ & $215(32.8)$ & $403(35.4)$ & 0.043 \\
\hline \multicolumn{6}{|l|}{ Anatomical disruption } \\
\hline Not present & $615(23.5)$ & $186(22.5)$ & $166(25.3)$ & $263(23.1)$ & 0.413 \\
\hline Yes, with localized peritonitis & $981(37.4)$ & $342(41.3)$ & $256(39.0)$ & $383(33.7)$ & 0.002 \\
\hline Yes, with diffuse peritonitis & $1025(39.1)$ & $300(36.2)$ & $234(35.7)$ & $491(43.2)$ & 0.001 \\
\hline
\end{tabular}

Data are reported as $n$ (\%) or median (1st-3rd quartile)

SAPS simplified acute physiology score, SOFA sequential organ failure assessment

${ }^{*} p$ value indicates differences between patients with community-acquired infection, healthcare-associated infection or early onset hospital-acquired infection, and late-onset hospital-acquired infection

**Data missing from 29 patients

***More details regarding underlying conditions are reported in Supplement-4 
Table 2 Proportion of types of intra-abdominal infection and distribution according to origin of infection acquisition

\begin{tabular}{lclcc} 
Type of abdominal sepsis & Total $\boldsymbol{n ( \% ) ^ { * }}$ & $\begin{array}{l}\text { Community-acquired } \\
\boldsymbol{n}(\%)^{* *}\end{array}$ & $\begin{array}{l}\text { Early onset hospital- } \\
\text { acquired } \boldsymbol{n}(\%)^{* *}\end{array}$ & $\begin{array}{l}\text { Late-onset } \\
\text { hospital-acquired } \\
\boldsymbol{n}(\%)^{* *}\end{array}$ \\
\hline Primary peritonitis & $103(3.9)$ & $33(32)$ & $28(27.2)$ & $42(40.8)$ \\
Secondary and tertiary peritonitis & $1794(68.4)$ & $588(32.8)$ & $431(24)$ & $775(43.2)$ \\
PD-related peritonitis & $9(0.3)$ & 0 & $2(20)$ & $7(70)$ \\
Intra-abdominal abscess & $180(6.9)$ & $36(20)$ & $49(27.2)$ & $95(52.8)$ \\
Biliary tract infection & $319(12.2)$ & $117(36.7)$ & $35(29.8)$ & $107(33.5)$ \\
Pancreatic infection & $165(6.3)$ & $45(27.3)$ & $3(33.3)$ & $87(52.7)$ \\
Typhlitis & $9(0.3)$ & 0 & $15(35.7)$ & $6(66.6)$ \\
Toxic megacolon & $42(1.6)$ & $9(21.4)$ & & $18(42.9)$ \\
\hline
\end{tabular}

$P D$-related peritoneal dialysis-related

*\% Within column; **\% within row

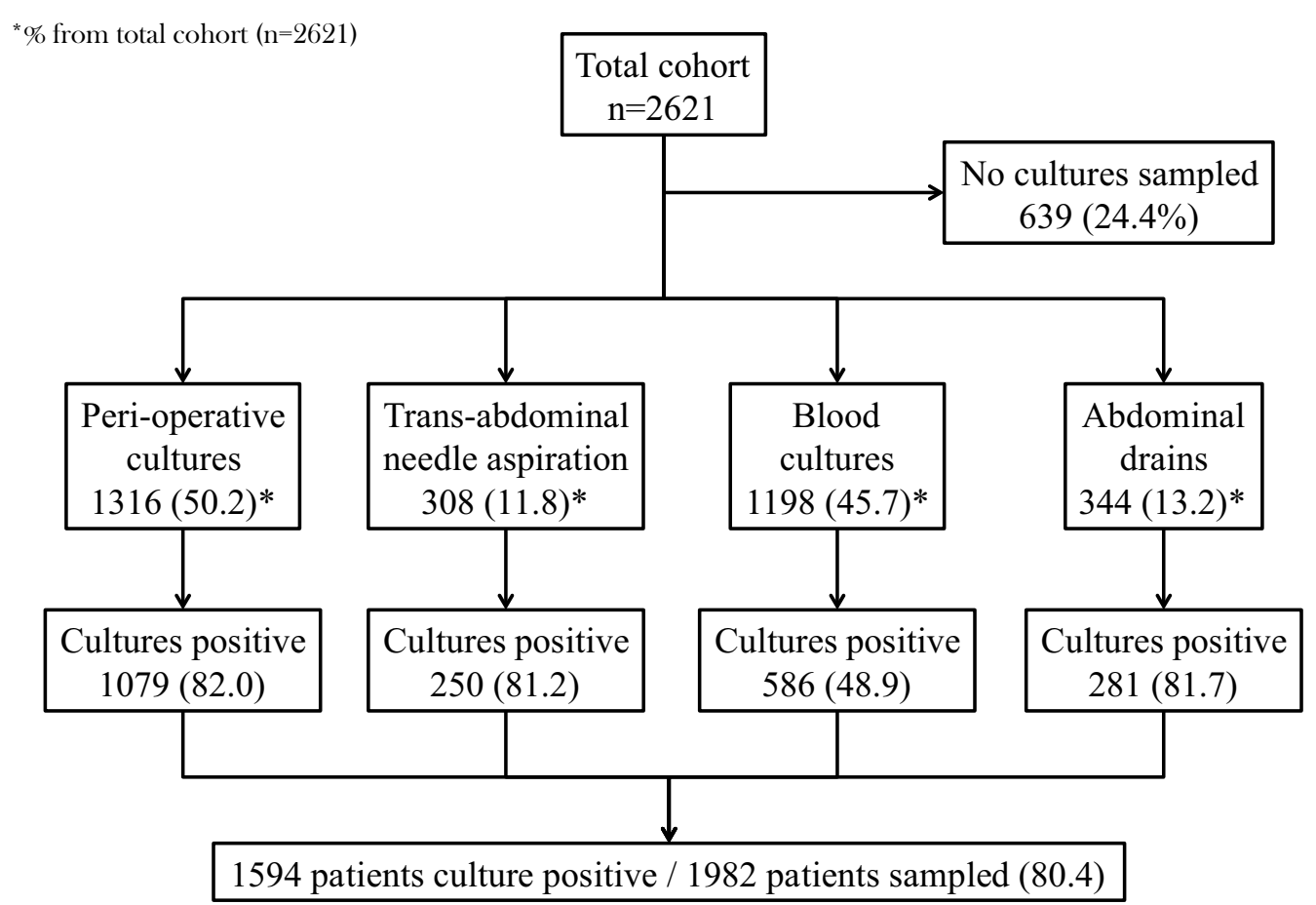

Fig. 1 Types of microbiological cultures sampled and culture-positive rate in patients with intra-abdominal infection

or carbapenem resistance, this covariate became significantly associated with mortality (Supplement-9).

\section{Discussion}

This multicenter observational study provided epidemiological insights in critically ill patients with intra-abdominal infection. The multicentre input of sequential cases of intra-abdominal infection offers a global view of the case mix of different presentations of intra-abdominal infection requiring ICU admission or occurring within the framework of an ICU stay. In spite of clinical heterogeneity, the core characteristics of intra-abdominal infection are quite generic including anatomical disruption and polymicrobial infection. Because of the broad variety in intra-abdominal infections, data were described according to a new classification based on setting of acquisition, presence of anatomical disruption, and severity of disease. Irrespective of type of 
Table 3 Micro-organisms isolated from cultures sampled in patients with intra-abdominal infection

\begin{tabular}{|c|c|c|c|c|}
\hline \multirow[t]{2}{*}{ Micro-organism } & \multirow{2}{*}{$\begin{array}{l}\text { Total cohort } \\
(n=1982)\end{array}$} & \multicolumn{3}{|c|}{ Setting of infection acquisition } \\
\hline & & $\begin{array}{l}\text { Community-acquired } \\
(n=664)\end{array}$ & $\begin{array}{l}\text { Early onset hospital- } \\
\text { acquired ( } n=482)\end{array}$ & $\begin{array}{l}\text { Late-onset } \\
\text { hospital-acquired } \\
(n=836)\end{array}$ \\
\hline Gram-negative bacteria & $1161(58.6)$ & $385(58)$ & $287(59.5)$ & $498(58.5)$ \\
\hline Enterobacterales & $1024(51.7)$ & $344(51.8)$ & $247(51.2)$ & $433(51.8)$ \\
\hline Citrobacter sp. & $21(1.1)$ & $6(0.9)$ & $8(1.7)$ & $7(0.8)$ \\
\hline Citrobacter freundii & $18(0.9)$ & $6(0.9)$ & $3(0.6)$ & $9(0.9)$ \\
\hline Escherichia coli & $729(36.8)$ & $252(38)$ & $172(35.7)$ & $304(36.4)$ \\
\hline Enterobacter aerogenes & $37(1.9)$ & $15(2.3)$ & $6(1.2)$ & $16(1.9)$ \\
\hline Enterobacter cloacae & $80(4)$ & $31(4.7)$ & $16(3.3)$ & $34(4.1)$ \\
\hline Hafnia alvei & $8(0.4)$ & $3(0.5)$ & $2(0.4)$ & $3(0.4)$ \\
\hline Morganella morganii & $25(1.3)$ & $10(1.5)$ & $5(1)$ & $10(1.2)$ \\
\hline Klebsiellasp. & $51(2.6)$ & $22(3.3)$ & $12(2.5)$ & $17(2)$ \\
\hline Klebsiella oxytoca* & $44(2.2)$ & $23(3.5)$ & $11(2.3)$ & $10(1.2)$ \\
\hline Klebsiella pneumoniae & $170(8.6)$ & $57(8.6)$ & $37(7.7)$ & $76(9.1)$ \\
\hline Proteus sp. & $23(1.2)$ & $9(1.4)$ & $7(1.5)$ & $7(0.8)$ \\
\hline Proteus mirabilis & $63(3.2)$ & $28(4.2)$ & $15(3.1)$ & $20(2.4)$ \\
\hline Providencia sp. & $3(0.2)$ & 0 & $1(0.2)$ & $2(0.2)$ \\
\hline Salmonella enterica & $4(0.2)$ & $2(0.3)$ & $2(0.4)$ & 0 \\
\hline Serratia marcescens & $12(0.6)$ & $2(0.3)$ & $4(0.8)$ & $6(0.7)$ \\
\hline Enterobacterales, other & $24(1.2)$ & $7(1.1)$ & $5(1)$ & $12(1.4)$ \\
\hline Non-fermenting bacteria & $233(11.8)$ & $72(10.8)$ & $66(13.7)$ & $95(11.4)$ \\
\hline Pseudomonas aeruginosa & $131(6.6)$ & $41(6.2)$ & $34(7.1)$ & $56(6.7)$ \\
\hline Pseudomonas sp. (other or NI) & $15(0.8)$ & $3(0.5)$ & $4(0.8)$ & $8(1)$ \\
\hline Stenotrophomonas maltophilia & $11(0.6)$ & $5(0.8)$ & $2(0.4)$ & $4(0.5)$ \\
\hline Acinetobacter baumannii & $61(6.2)$ & $18(2.7)$ & $22(4.6)$ & $21(2.5)$ \\
\hline Acinetobacter sp. (other or NI) & $32(1.6)$ & $8(1.2)$ & $12(2.5)$ & $12(1.4)$ \\
\hline \multicolumn{5}{|l|}{ Other Gram-negative bacteria } \\
\hline Haemophilus influenzae & $4(0.2)$ & $2(0.3)$ & 0 & $2(0.2)$ \\
\hline Gram-positive bacteria & $781(39.4)$ & $274(41.3)$ & $187(38.8)$ & $320(38.3)$ \\
\hline Staphylococci & $195(9.8)$ & $69(10.4)$ & $44(9.1)$ & $82(9.8)$ \\
\hline Staphylococcus aureus & $64(3.2)$ & $23(3.5)$ & $19(3.9)$ & $22(2.6)$ \\
\hline Coagulase-negative staphylococci & $100(5)$ & $37(5.6)$ & $23(4.8)$ & $40(4.8)$ \\
\hline Staphylococcus sp. (other or NI) & $37(1.9)$ & $11(1.7)$ & $5(1)$ & $21(2.5)$ \\
\hline Enterococci & $513(25.9)$ & $173(26.1)$ & $121(25.1)$ & $219(26.2)$ \\
\hline Enterococcus faecalis & $257(13)$ & $83(12.5)$ & $59(12.2)$ & $115(13.8)$ \\
\hline Enterococcus faecium & $216(10.9)$ & $70(10.5)$ & $46(9.5)$ & $100(12)$ \\
\hline Enterococcus sp. (other or NI) & $77(3.9)$ & $33(5)$ & $18(3.7)$ & $26(3.1)$ \\
\hline \multicolumn{5}{|l|}{ Other Gram-positive bacteria } \\
\hline Streptococcus Group A, B, C, G & $117(5.9)$ & $44(6.6)$ & $27(5.6)$ & $46(5.5)$ \\
\hline Streptococcus pneumoniae & $9(0.5)$ & $4(0.6)$ & $2(0.4)$ & $3(0.4)$ \\
\hline Streptococcus viridans & $33(1.7)$ & $13(2)$ & $7(1.5)$ & $13(1.6)$ \\
\hline Corynebacterium & $8(0.4)$ & $1(0.2)$ & $3(0.6)$ & $4(0.5)$ \\
\hline Anaerobe bacteria & $231(11.7)$ & $83(12.5)$ & $45(9.3)$ & $103(12.3)$ \\
\hline Clostridium perfringens & $21(1.1)$ & $7(1.1)$ & $3(0.6)$ & $11(1.3)$ \\
\hline Peptostreptococcus sp. & $4(0.2)$ & $1(0.2)$ & $2(0.4)$ & $1(0.1)$ \\
\hline Actinomyces sp. & $2(0.1)$ & $1(0.2)$ & 0 & $1(0.1)$ \\
\hline Gram-positive anaerobe sp. (other or NI) & $53(2.7)$ & $17(2.6)$ & $12(2.5)$ & $24(2.9)$ \\
\hline Clostridium difficile & $8(0.4)$ & $3(0.5)$ & $1(0.2)$ & $4(0.5)$ \\
\hline Bacteroides sp.* & $103(5.2)$ & $46(6.9)$ & $17(3.5)$ & $40(4.8)$ \\
\hline
\end{tabular}


Table 3 (continued)

\begin{tabular}{|c|c|c|c|c|}
\hline \multirow[t]{2}{*}{ Micro-organism } & \multirow{2}{*}{$\begin{array}{l}\text { Total cohort } \\
(n=1982)\end{array}$} & \multicolumn{3}{|c|}{ Setting of infection acquisition } \\
\hline & & $\begin{array}{l}\text { Community-acquired } \\
(n=664)\end{array}$ & $\begin{array}{l}\text { Early onset hospital- } \\
\text { acquired }(n=482)\end{array}$ & $\begin{array}{l}\text { Late-onset } \\
\text { hospital-acquired } \\
(n=836)\end{array}$ \\
\hline Porphyromonas sp. & $2(0.1)$ & 0 & $2(0.4)$ & 0 \\
\hline Prevotella sp. & $5(0.3)$ & $3(0.5)$ & 0 & $2(0.2)$ \\
\hline Fusobacterium sp. & $9(0.5)$ & $7(1.1)$ & 0 & $2(0.2)$ \\
\hline Gram-negative anaerobe sp. (other or NI) & $66(3.3)$ & $20(3)$ & $13(2.7)$ & $33(3.9)$ \\
\hline Fungi & $258(13)$ & $80(12)$ & $71(14.7)$ & $107(12.8)$ \\
\hline Aspergillus sp. & $3(0.2)$ & 0 & $2(0.4)$ & $1(0.1)$ \\
\hline Candida sp. & $257(13)$ & $81(12.2)$ & $69(14.3)$ & $107(12.8)$ \\
\hline Candida albicans & $173(8.7)$ & $56(8.4)$ & $50(10.4)$ & $67(8)$ \\
\hline Candida glabrata & $35(1.8)$ & $10(1.5)$ & $9(1.9)$ & $16(1.9)$ \\
\hline Candida krusei & $3(0.2)$ & $2(0.3)$ & 0 & $1(0.1)$ \\
\hline Candida parapsilosis & $9(0.5)$ & $4(0.6)$ & $1(0.2)$ & $4(0.5)$ \\
\hline Candida tropicalis & $16(0.8)$ & $6(0.9)$ & $2(0.4)$ & $8(1)$ \\
\hline Candida sp. (other or NI) & $20(1)$ & $2(0.3)$ & $7(1.5)$ & $11(1.3)$ \\
\hline
\end{tabular}

Table reports $n$ patients positive (\% of total number of patients with cultures sampled)

$N /$ not identified

${ }^{*} p<0.05$ for differences between setting of infection acquisition

intra-abdominal infection, mortality was higher in lateonset hospital-acquired cases with diffuse peritonitis and septic shock. This classification allows comparison across a spectrum of intra-abdominal infections and might be used for including patients in future clinical trials.

There were no differences in the prevalence of antimicrobial resistance in microbiological cultures sampled in community-acquired vs. early onset vs. late-onset hospital-acquired infection. This may be explained at least in part by the spread of resistance clones/genes into the community, as is the case for ESBL-producing or carbapenem-resistant Enterobacterales (formerly known as Enterobacteriaceae). This is certainly the case for risk regions such as Eastern and South-East Europe, the Middle-East, and Latin America, and matches with the results of a global point prevalence study on antimicrobial consumption and resistance [17]. This confirms the trend that classic risk factors for antimicrobial resistance involvement are losing predictive value as illustrated in a multicenter study reporting antimicrobial resistance in $39 \%$ of infections in patients without an obvious risk profile as evidenced by prior antibiotic exposure and/or hospitalisation [18]. This observation is highly relevant as it might stress the need for last-line antimicrobial therapy in community-acquired infection in selected regions. Considering local ecology together with the individual patient profile, and disease severity remains essential. However, antimicrobial resistance in key-pathogens isolated in intra-abdominal infection does not seem to be associated with increased virulence, as it occurred at similar rates in infection, sepsis, and septic shock. Overall prevalence of enterococci was $26 \%$ and thereby substantially higher as previously reported [19-22]. This trend can be attributed to the steadily emergence of enterococci in acute care settings or to the particular composition of a cohort of exclusively critically ill patients [23].

No differences in empiric antibacterial regimens were observed according to setting of infection acquisition. Anti-pseudomonal coverage was provided up-front in not only late-onset cases, a supposed classic risk factor for antimicrobial resistant infection, including $P$. aeruginosa strains, but also in community-acquired or early onset hospital-acquired infections. This is probably triggered by a safety-reflex in physicians, not to miss any potential pathogen, especially $P$. aeruginosa strains. Thus, the risk factor-based antibiotic strategy that appears in all guidelines seems not to be implemented in a large real-life sample of intra-abdominal infection in the ICU, reflecting response to severity.

It is reassuring that the vast majority of intra-abdominal infections in the ICU were approached by an early source control intervention. It has been established that surgery needs to be performed after hemodynamic stabilization, but nevertheless should be performed as early as possible aiming at damage control [24]. The importance of source is evidenced by the increased mortality among patients with persistent inflammation or need for additional surgical intervention. 
Table 4 Rates of antimicrobial resistance in intra-abdominal infections according to geographic region

\begin{tabular}{|c|c|c|c|c|c|c|c|c|c|}
\hline \multirow{2}{*}{$\begin{array}{l}\text { Antibiotic- } \\
\text { resistant } \\
\text { pathogen }\end{array}$} & \multirow{2}{*}{$\begin{array}{l}\text { Total } \\
\text { cohort } \\
(n=1982)\end{array}$} & \multicolumn{8}{|c|}{ Geographic region } \\
\hline & & $\begin{array}{l}\text { Western } \\
\text { Europe } \\
(n=601)\end{array}$ & $\begin{array}{l}\text { Southern } \\
\text { Europe } \\
(n=558)\end{array}$ & $\begin{array}{l}\text { Eastern } \\
\text { and South- } \\
\text { East Europe } \\
(n=151)\end{array}$ & $\begin{array}{l}\text { Central } \\
\text { Europe } \\
(n=99)\end{array}$ & $\begin{array}{l}\text { North Africa } \\
\text { and Mid- } \\
\text { dle-East } \\
(n=172)\end{array}$ & $\begin{array}{l}\text { Latin } \\
\text { America } \\
(n=249)\end{array}$ & $\begin{array}{l}\text { North } \\
\text { America } \\
(n=22)\end{array}$ & $\begin{array}{l}\text { Asia-Pacific } \\
(n=123)\end{array}$ \\
\hline $\begin{array}{l}\text { Difficult-to- } \\
\text { treat resist- } \\
\text { ant Gram- } \\
\text { negative } \\
\text { bacteria }\end{array}$ & $85(4.3)$ & $2(0.3)$ & $38(6.8)$ & $9(6)$ & 0 & $15(8.7)$ & $16(6.4)$ & 0 & $5(4.1)$ \\
\hline $\begin{array}{l}\text { Any resistant } \\
\text { Gram- } \\
\text { negative } \\
\text { bacteria* }\end{array}$ & $480(24.2)$ & $54(9)$ & $140(25.1)$ & $59(39.1)$ & $20(20.2)$ & $82(47.7)$ & $90(36.1)$ & $7(31.8)$ & $26(21.1)$ \\
\hline $\begin{array}{l}\text { ESBL- } \\
\text { producing } \\
\text { Gram- } \\
\text { negative } \\
\text { bacteria }\end{array}$ & $326(16.4)$ & $37(6.2)$ & $81(14.5)$ & $37(24.5)$ & $9(9.1)$ & 65 (37.8) & $69(27.7)$ & $7(31.8)$ & $20(16.3)$ \\
\hline $\begin{array}{l}\text { Carbap- } \\
\text { enem- } \\
\text { resistant } \\
\text { Gram- } \\
\text { negative } \\
\text { bacteria }\end{array}$ & $145(7.3)$ & $3(0.5)$ & $61(10.9)$ & $23(15.2)$ & $1(1)$ & $23(13.4)$ & $25(10)$ & 0 & $9(7.3)$ \\
\hline $\begin{array}{c}\text { Fluoroqui- } \\
\text { nolone- } \\
\text { resistant } \\
\text { Gram- } \\
\text { negative } \\
\text { bacteria }\end{array}$ & $339(17.1)$ & $29(4.8)$ & $108(19.4)$ & $37(24.5)$ & $18(18.2)$ & $57(33.1)$ & $69(27.7)$ & $3(13.6)$ & $17(13.8)$ \\
\hline MRSA & $20(1)$ & $1(0.2)$ & $5(0.9)$ & $5(3.3)$ & 0 & $5(2.9)$ & $3(1.2)$ & 0 & $1(0.8)$ \\
\hline VRE & $56(2.8)$ & $11(1.8)$ & $15(2.7)$ & $5(3.3)$ & $2(2)$ & $9(5.2)$ & $11(4.4)$ & $1(4.5)$ & $2(1.6)$ \\
\hline $\begin{array}{l}\text { Antimicrobial } \\
\text { resistance** } \\
\text { (total) }\end{array}$ & $153(7.7)$ & $14(2.3)$ & $57(10.2)$ & $16(10.6)$ & $2(2)$ & $29(16.9)$ & $27(10.8)$ & $1(4.5)$ & $7(5.7)$ \\
\hline $\begin{array}{l}\text { Antimicrobial } \\
\text { resist- } \\
\text { ance*** } \\
\text { (total) }\end{array}$ & $522(26.3)$ & $63(10.5)$ & $152(27.2)$ & $65(43)$ & $21(21.2)$ & $87(50.6)$ & 96 (38.6) & $8(36.4)$ & $28(22.8)$ \\
\hline
\end{tabular}

\% Represent proportion per column; Resistance rates reflect proportion of patients in which resistant strains are isolated (e.g., $n$ MRSA/total $n$ patients) and do not represent proportion of resistance within particular pathogens (e.g., $n$ MRSA/total S. aureus isolates)

Denominator for microbiological data includes only patients in which cultures were sampled (data from South Africa are excluded as they included only seven patients)

ESBL extended-spectrum beta-lactamase-producing, MRSA methicillin-resistant Staphylococcus aureus, VRE vancomycin-resistant enterococci

*Gram-negative bacteria that are either ESBL-producing, or carbapenem-resistant, or fluoroquinolone-resistant

**Total rates of multidrug resistance considering difficult-to-treat resistant Gram-negative bacteria, MRSA, and VRE

***Total rates of multidrug resistance considering any type of Gram-negative resistance (either ESBL-producing, or carbapenem-resistant, or fluoroquinolone-resistant bacteria), MRSA, and VRE

Late-onset hospital-acquired infection, diffuse peritonitis, and septic shock were identified as independent risk factors for mortality, and confirm the robustness of the new classification system for risk stratification. Antimicrobial resistance defined as either MRSA, VRE, ESBLproducing, or carbapenem-resistant Gram-negative bacteria was independently associated with increased mortality (Supplement-9). Surprisingly, however, the more strict definition of either MRSA, VRE, or difficultto-treat resistant Gram-negative bacteria was not associated with increased mortality. Probably, the cohort lacked sufficient power as in only 85 patients, difficult-to-treat Gram-negatives were involved vs. 341 ESBL-producing or carbapenem-resistant Gram-negative bacteria. We have no explanation for the favorable association with antiMRSA agents. This can hardly be due to the anti-MRSA 


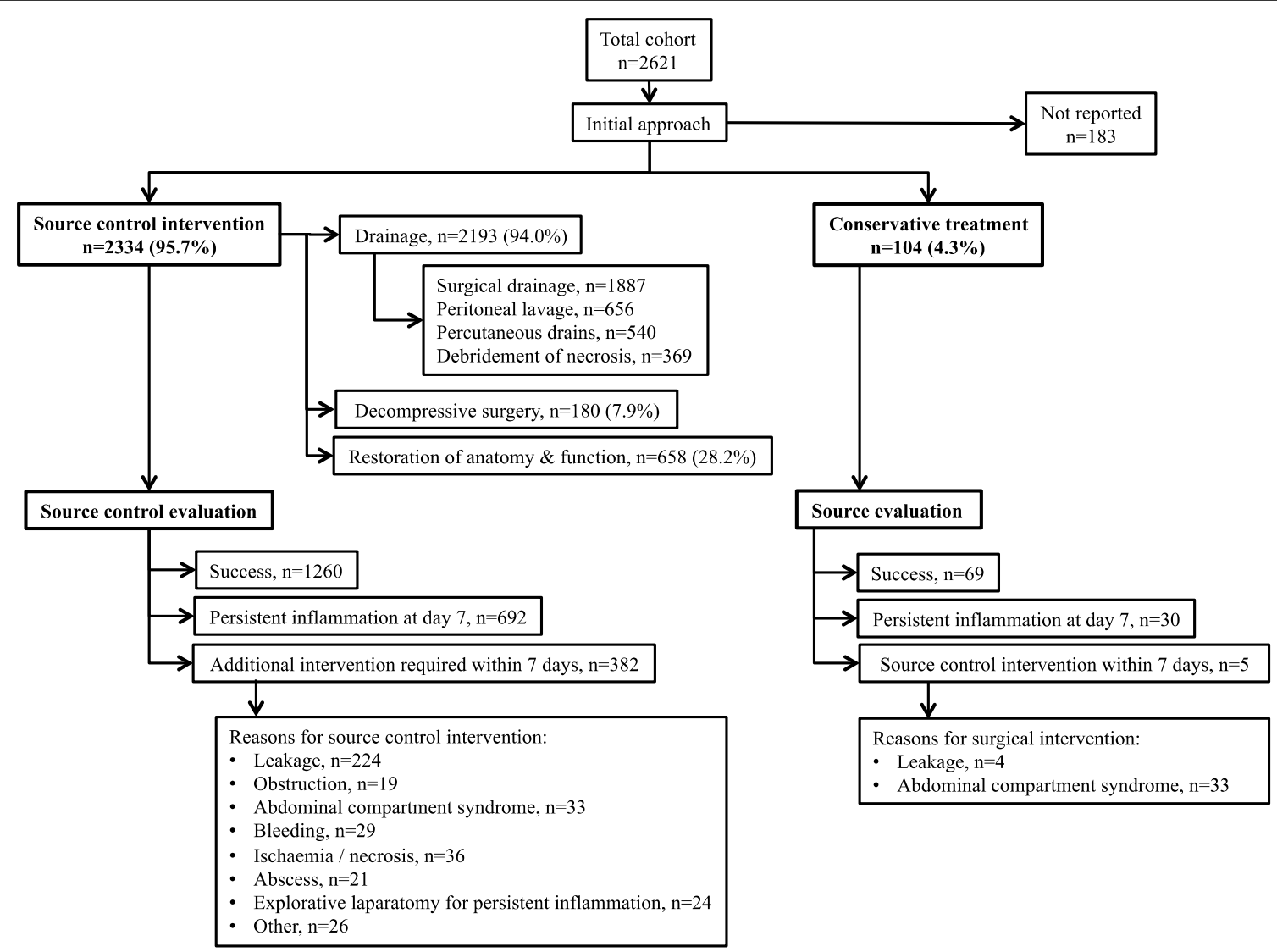

Legend: Several types of source control interventions could have been executed in a single patient.

Fig. 2 Initial approach to control the source of infection. Several types of source control interventions could have been executed in a single patient

Table 5 Mortality according to alternative classification of intra-abdominal infection

\begin{tabular}{|c|c|c|c|c|c|c|c|c|c|}
\hline \multirow{3}{*}{$\begin{array}{l}\begin{array}{l}\text { Severity } \\
\text { of disease } \\
\text { expression }\end{array} \\
\text { Septic shock }\end{array}$} & \multicolumn{9}{|c|}{ Setting of infection acquisition } \\
\hline & \multicolumn{3}{|c|}{ Community-acquired } & \multicolumn{3}{|c|}{ Early onset hospital-acquired } & \multicolumn{3}{|c|}{ Late-onset hospital-acquired } \\
\hline & $\begin{array}{l}18 / 64 \\
28.1 \%\end{array}$ & $\begin{array}{l}25 / 83 \\
30.1 \%\end{array}$ & $\begin{array}{l}48 / 101 \\
47.5 \%\end{array}$ & $\begin{array}{l}21 / 63 \\
33.3 \%\end{array}$ & $\begin{array}{l}13 / 61 \\
21.3 \%\end{array}$ & $\begin{array}{l}37 / 91 \\
40.7 \%\end{array}$ & $\begin{array}{l}45 / 103 \\
43.7 \%\end{array}$ & $\begin{array}{l}48 / 110 \\
43.6 \%\end{array}$ & $\begin{array}{l}94 / 190 \\
49.5 \%\end{array}$ \\
\hline Sepsis & $\begin{array}{l}13 / 116 \\
11.2 \%\end{array}$ & $\begin{array}{l}42 / 221 \\
19 \%\end{array}$ & $\begin{array}{l}37 / 174 \\
21.3 \%\end{array}$ & $\begin{array}{l}27 / 90 \\
30 \%\end{array}$ & $\begin{array}{l}33 / 170 \\
19.4 \%\end{array}$ & $\begin{array}{l}43 / 128 \\
33.6 \%\end{array}$ & $\begin{array}{l}26 / 147 \\
17.7 \%\end{array}$ & $\begin{array}{l}62 / 237 \\
26.2 \%\end{array}$ & $\begin{array}{l}99 / 275 \\
36 \%\end{array}$ \\
\hline \multirow[t]{3}{*}{ Infection } & $\begin{array}{l}1 / 7 \\
14.3 \%\end{array}$ & $\begin{array}{l}3 / 22 \\
13.6 \%\end{array}$ & $\begin{array}{l}4 / 22 \\
18.2 \%\end{array}$ & $\begin{array}{l}0 / 7 \\
0 \%\end{array}$ & $\begin{array}{l}0 / 21 \\
0 \%\end{array}$ & $\begin{array}{l}2 / 14 \\
14.3 \%\end{array}$ & $\begin{array}{l}1 / 12 \\
8.3 \%\end{array}$ & $\begin{array}{l}8 / 36 \\
22.2 \%\end{array}$ & $\begin{array}{l}2 / 23 \\
8.7 \%\end{array}$ \\
\hline & No & $\begin{array}{l}\text { Yes, with } \\
\text { localized } \\
\text { peritonitis }\end{array}$ & $\begin{array}{l}\text { Yes, with } \\
\text { diffuse } \\
\text { peritonitis }\end{array}$ & No & $\begin{array}{l}\text { Yes, with } \\
\text { localized } \\
\text { peritonitis }\end{array}$ & $\begin{array}{l}\text { Yes, with } \\
\text { diffuse } \\
\text { peritonitis }\end{array}$ & No & $\begin{array}{l}\text { Yes, with } \\
\text { localized } \\
\text { peritonitis }\end{array}$ & $\begin{array}{l}\text { Yes, with } \\
\text { diffuse } \\
\text { peritonitis }\end{array}$ \\
\hline & \multicolumn{3}{|c|}{ Anatomical disruption } & \multicolumn{3}{|c|}{ Anatomical disruption } & \multicolumn{3}{|c|}{ Anatomical disruption } \\
\hline
\end{tabular}

activity as such, since MRSA was isolated in only 20 patients. The advantageous association might be due to the anti-enterococcal activity of these agents. Yet, enterococcal coverage as such (not necessarily covering MRSA) was not retained in the final regression model assessing relationships with mortality. Hence, this observation might just be an incidental finding. On the other hand, the absence of an association between empiric antifungal therapy and outcome seems consistent with the finding of other cohort studies and randomized-controlled trials that did not demonstrate the effect of empirical Candida coverage and favorable outcome $[25,26]$. 
Table 6 Independent relationships with mortality in critically ill patients with intra-abdominal infection

\begin{tabular}{|c|c|c|}
\hline Variable & $\begin{array}{l}\text { Model with source control achievement* } \\
\text { OR }(95 \% \mathrm{Cl})\end{array}$ & $\begin{array}{l}\text { Model without source } \\
\text { control achievement** } \\
\text { OR }(95 \% \mathrm{Cl})\end{array}$ \\
\hline \multicolumn{3}{|l|}{ Setting of infection acquisition } \\
\hline Community-acquired infection & Reference & Reference \\
\hline Early onset hospital-acquired infection ( $\leq 7$ days) & $1.15(0.84-1.58)$ & $1.18(0.88-1.59)$ \\
\hline Late-onset hospital-acquired infection (> 7 days) & $1.76(1.34-2.32)$ & $1.76(1.36-2.30)$ \\
\hline \multicolumn{3}{|l|}{ Anatomical disruption } \\
\hline No anatomical barrier disruption & Reference & Reference \\
\hline Anatomical disruption with localized peritonitis & $1.28(0.95-1.75)$ & $1.26(0.95-1.69)$ \\
\hline Anatomical disruption with diffuse peritonitis & $1.99(1.49-2.67)$ & $2.04(1.55-2.70)$ \\
\hline \multicolumn{3}{|l|}{ Severity of disease expression } \\
\hline Infection & Reference & Reference \\
\hline Sepsis & $2.44(1.37-4.66)$ & $2.28(1.31-4.28)$ \\
\hline Septic shock & $5.22(2.91-10)$ & $4.93(2.80-9.30)$ \\
\hline Age (per year increase) & $1.03(1.02-1.04)$ & $1.03(1.03-1.04)$ \\
\hline \multicolumn{3}{|l|}{ Underlying conditions } \\
\hline Malnutrition (body mass index<20) & $2.07(1.34-3.17)$ & $2.15(1.43-3.21)$ \\
\hline Diabetes mellitus & $1.31(0.99-1.73)$ & $1.32(1.01-1.72)$ \\
\hline Liver failure & $2.03(1.23-3.33)$ & $2.50(1.55-4.02)$ \\
\hline Congestive heart failure & $1.86(1.24-2.81)$ & $1.92(1.31-2.81)$ \\
\hline \multicolumn{3}{|l|}{ Empiric antimicrobial coverage } \\
\hline Anti-MRSA agent & $0.77(0.59-1)$ & $0.77(0.59-0.98)$ \\
\hline Double anaerobe coverage & - & $1.28(0.97-1.71)$ \\
\hline \multicolumn{3}{|l|}{ Source control achievement at day 7} \\
\hline Success & Reference & - \\
\hline Failure, persistent signs of inflammation & $4.85(3.79-6.22)$ & - \\
\hline Failure, additional intervention required following initial approach & $1.93(1.41-2.65)$ & - \\
\hline
\end{tabular}

The variable "antimicrobial resistance" defined as either MRSA, vancomycin-resistant enterococci (VRE), or difficult-to-treat resistant Gram-negative bacteria did not achieve the final regression model. Supplement-9 reports the results of the logistic regression models with antibiotic resistance defined as either MRSA, VRE, ESBLproducing, or carbapenem-resistant Gram-negative bacteria. In these logistic regression models, antibiotic resistance was associated with increased risk of mortality, while other covariates remained stable

OR odds ratio, $\mathrm{Cl}$ confidence interval, MRSA methicillin-resistant Staphylococcus aureus

*Area under the receiver-operating curve characteristic: $0.778 ;{ }^{* *}$ Area under the receiver-operating curve characteristic: 0.689

This study has limitations. This is an observational cohort study disposed to confounding. Some geographic regions are poorly represented obstructing conclusive results. Evaluation of source control achievement remains a subjective appreciation performed by the attending physician; given the study scale, it was not feasible to establish an independent panel for in-depth evaluation of source control as previously reported [27]. At the same line, given the observational study design, there was no predefined approach to source control [7]. In addition, with this paper, we intended to provide a general epidemiological snapshot. Therefore, detailed country-specific or disease-specific analyses fell outside the scope of this report. Finally, we could not report the proportion of ICU patients with intra-abdominal infection/sepsis as the total number of admissions during the inclusion of cases was not recorded.
In conclusion, this multinational cohort of ICU patients with intra-abdominal infection revealed that late-onset healthcare-associated infection, diffuse peritonitis, and sepsis or septic shock are independent risk factors for mortality. Therefore, setting of infection acquisition, anatomical disruption, and severity of disease expression are disease-specific phenotypic characteristics associated with outcome, irrespective of the type of intra-abdominal infection. Antimicrobial resistance is mainly an issue of Gram-negatives and a particular concern in specific geographic areas and associated with worse outcome as was failure of source control.

\section{Electronic supplementary material}

The online version of this article (https://doi.org/10.1007/s00134-019-05819-3) contains supplementary material, which is available to authorized users. 


\section{Author details}

${ }^{1}$ Department of Internal Medicine and Pediatrics, Ghent University, Campus UZ Gent, Corneel Heymanslaan 10, 9000 Ghent, Belgium. ${ }^{2}$ Department of Anesthesiology, Intensive Care and Emergency Medicine, Fondazione Policlinico Universitario A. Gemelli IRCCS, Rome, Italy. ${ }^{3}$ Università Cattolica del Sacro Cuore, Rome, Italy. ${ }^{4}$ Intensive Care Unit, Papageorgiou University Affiliated Hospital, Thessaloníki, Greece. ${ }^{5}$ Surrey Perioperative Anaesthetic Critical Care Collaborative Research Group (SPACeR), Royal Surrey County Hospital, Guildford, UK. ${ }^{6}$ Department of Clinical and Experimental Medicine, University of Surrey, Guildford, UK. ${ }^{7}$ Department of Intensive Care Medicine, University Medical Center Utrecht, University Utrecht, Utrecht, The Netherlands. ${ }^{8}$ Department of Critical Care Medicine, Ghent University Hospital, Ghent, Belgium. ${ }^{9}$ Strategic Policy Cell, Ghent University Hospital, Ghent, Belgium. ${ }^{10}$ Department of Anesthesiology and Reanimation, Cerrahpasa School of Medicine, Istanbul University-Cerrahpasa, Istanbul, Turkey. ${ }^{11}$ Critical Care Department, University Hospital ATTIKON, National and Kapodistrian University of Athens, Athens, Greece. ${ }^{12}$ Department of General, Visceral and Thoracic Surgery, Klinikum Peine, Medical University Hannover, Hannover, Germany. ${ }^{13}$ Division of Scientific Affairs-Research, European Society of Intensive Care Medicine, Brussels, Belgium. ${ }^{14}$ Anesthesia and Intensive Care Department, University Hospital of Modena, Modena, Italy. ${ }^{15}$ Burns, Trauma and Critical Care Research Centre, Centre for Clinical Research, Faculty of Medicine, The University of Queensland, Brisbane, Australia. ${ }^{16}$ 2nd Critical Care Department, Attikon University Hospital, Athens, Greece. ${ }^{17}$ Department of Nursing, Faculty of Education, Health and Social Work, University College Ghent, Ghent, Belgium. ${ }^{18}$ Royal Brisbane and Women's Hospital, The University of Queensland, Brisbane, Australia. ${ }^{19}$ Nimes University Hospital, University of Montpellier, Nimes, France. ${ }^{20}$ Critical Care Department, Hospital of the Interamerican Open University (UAI), Buenos Aires, Argentina. ${ }^{21}$ Surgical Critical Care, Department of Anesthesia, Hospital Universitario La Paz-IdiPaz, Madrid, Spain. ${ }^{22}$ Université de Paris, INSERM, UMR 1152, Paris, France. ${ }^{23}$ Anesthesiology and Critical Care Medicine, Bichat-Claude Bernard University Hospital, HUPNSV, AP-HP, Paris, France. ${ }^{24}$ Faculty of Health Sciences, Poznan University of Medical Sciences, Poznan, Poland. ${ }^{25}$ Department of Anaesthesiology and Intensive Therapy, Regional Hospital in Poznan, Poznan, Poland. ${ }^{26}$ Intensive Care Department, Faculty of Medicine, Centro Hospitalar Universitario S. Joao, University of Porto, Grupo Infecçao e Sepsis, Porto, Portugal. ${ }^{27}$ Intensive Care Unit from Hospital Interzonal General de Agudos "Prof Dr Luis Guemes", Buenos Aires, Argentina. ${ }^{28}$ Ciberes and Vall d'Hebron Institute of Research, Barcelona, Spain. ${ }^{29}$ Université de Paris, IAME, INSERM, Paris 75018, France. ${ }^{30}$ AP-HP, Hôpital Bichat, Medical and Infection Diseases ICU (MI2), Paris 75018, France. ${ }^{31}$ General Internal Medicine, Infectious Diseases, and Psychometric Medicine, Ghent University Hospital, Ghent, Belgium

\section{Acknowledgements \\ Collaborators AbSeS study: National Coordinators: Algeria: Amin} Lamrous (CHU Alger), Argentina: Cecilia Pereyra (Hospital Interzonal Agudos Prof Dr Luis Guemes, Buenos Aires), Fernando Lipovestky (Universidad Abierta Interamericana Hospital, Buenos Aires); Australia: Despoina Koulenti (UQCCR, Faculty of Medicine, The University of Queensland, Brisbane); Belgium: Jan De Waele (Ghent University Hospital, Ghent); Canada: Joao Rezende-Neto (St Michael's Hospital, Toronto); (Colombia: Yenny Cardenas (Hospital Universitario Fundación Santa Fe, Bogotá); Czech Republic: Tomas Vymazal (Motol University Hospital, Prague); Denmark: Hans Fjeldsoee-Nielsen (FjeldsoeeNielsen (Nykoebing Falster Hospital, Nykoebing Falster); France: Philippe Montravers (CHU Bichat Claude Bernard, Paris); Germany: Matthias Kott (Universitätsklinikum, Schleswig-Holstein, Kiel); Greece: Arvaniti Kostoula (Papageorgiou General Hospital, Thessaloniki); India: Yash Javeri (Nayati Healthcare, Delhi); Italy: Massimo Girardis (University Hospital of Modena, Modena); Israel: Sharon Einav (Shaare Zedek Medical Centre, Jerusalem); Netherlands: Dylan de Lange (University Medical Center, Utrecht); Peru: Luis Daniel Umezawa Makikado (Clínica Ricardo Palma, Lima); Poland: Adam Mikstacki (Regional Hospital, Poznan); Portugal: José-Artur Paiva (Centro Hospitalar Universitário Sao João, Porto); Romania: Dana Tomescu (Fundeni Clinical Institute, Bucharest); Russian Federation: Alexey Gritsan (Krasnoyarsk State Medical University, Krasnoyarsk Regional Clinical Hospital, Krasnoryarsk); Serbia; Bojan Jovanovic (Clinical Center of Serbia, Belgrade); Singapore: Kumaresh Venkatesan (Khoo Teck Puat Hospital, Singapore); Slovenia: Tomislav Mirkovic (University Medical Centre, Ljubljana); Spain: Emilio Maseda (Hospital Universitario La Paz, Madrid); Turkey: Yalim Dikmen (Istanbul University-Cerrahpasa, Cerrahpasa Medical School, Istanbul); United
Kingdom: Benedict Creagh-Brown (Royal Surrey County Hospital NHS Foundation Trust, Guilford); Investigators: ALGERIA: CHU (Algiers): Amin Lamrous; ARGENTINA: Sanatorio Güemes (Buenos Aires): Monica Emmerich, Mariana Canale; Sanatorio de la Trinidad Mitre (Buenos Aires): Lorena Silvina Dietz, Santiago llutovich; Hospital General de Agudos "Dr. Teodoro Alvarez" (Buenos Aires): John Thomas Sanchez Miñope, Ramona Baldomera Silva; Hospital Militar Central (Buenos Aires): Martin Alexis Montenegro, Patricio Martin; Policlinico Central Union Obrera Metalurgica (Buenos Aires): Pablo Saul, Viviana Chediack; Sanatorio San José (Buenos Aires): Giselle Sutton, Rocio Couce; Hospital General de Agudos "Dr. Ignacio Pirovano" (Buenos Aires): Carina Balasini, Susana Gonzalez; Hospital Britanico (Buenos Aires): Florencia Maria Lascar, Emiliano Jorge Descotte; CMPF Churruca-Visca (Buenos Aires): Natalia Soledad Gumiela, Carina Alejandra Pino; Clinica San Camilo (Buenos Aires): Cristian Cesio, Emanuel Valgolio; Hospital Francisco Javier Muñiz (Buenos Aires): Eleonora Cunto, Cecilia Dominguez; Universidad Abierta Interamericana Hospital (Buenos Aires): Fernando Lipovestky; Hospital Alberto Balestrini (Buenos Aires): Nydia Funes Nelson, Esteban Martin Abegao; Hospital Interzonal Agudos Prof Dr Luis Güemes (Buenos Aires): Cecilia Pereyra, Norberto Christian Pozo; Hospital Español (Buenos Aires): Luciana Bianchi, Enrique Correger; Clinica Zabala (Caba): Maria Laura Pastorino, Erica Aurora Miyazaki; Hospital César Milstein (Caba): Norberto Christian Pozo, Nicolas Grubissich; Hospital Regional Victor Sanguinetti (Comodoro): Mariel Garcia, Natalia Bonetto; Hospital Municipal de Urgencias (Cordoba): Noelia Elizabeth Quevedo, Cristina Delia Gomez; Hospital Manuel B Cabrera (Coronel Pringles): Felipe Queti, Luis Gonzalez Estevarena; Hospital Español de Mendoza (Mendoza,Godoy Cruz: Ruben Fernandez, Ignacio Santolaya; H.I.G.A. Prof. Dr. Luis Güemes (Haedo): Norberto Christian Pozo; Hospital Municipa Doctor Carlos Macias (Mar de Ajo): Sergio Hugo Grangeat, Juan Doglia; Hospital Luis C. Lagomaggiore (Mendoza): Graciela Zakalik, Carlos Pellegrini; Hospital Nacional Profesor Alejandro Posadas (Moron): Maria Monserrat Lloria, Mercedes Esteban Chacon; Hospital Provincial de Neuquen (Neuquen): Mariela Fumale; Clinica Modelo S.A (Paraná): Mariela Leguizamon; Sanatorio de la Ciudad (Puerto Madryn): Irene Beatriz Hidalgo, Roberto Julian Tiranti; Sanatorio Nosti (Rafaela): Paola Capponi, Agustin Tita; Hospital Provincial del Centenario (Rosario): Luis Cardonnet, Lisandro Bettini; Sanatorio Parque (Rosario): Agñel Ramos, Luciano Lovesio; Hospital Papa Francisco (Salta): Edith Miriam Miranda, Angelica Beatriz Farfan; Hospital San Juan Bautista (San Fernando del Valle de Catamarca): Carina Tolosa, Lise Segura; Hospital Central San Isidro Dr Melchor A. Posse (San Isidro-Buenos Aires): Adelina Bellocchio, Brian Alvarez; Hospital Guillermo Rawson (San Juan): Adriana Manzur, Rodolfo Lujan; Establecimiento Asistencial Dr Lucio Molas (Santa Rosa): Natalia Fernandez, Nahuel Scarone; Clínica de Especialidades (Villa María): Alan Zazu, Carina Groh; AUSTRALIA: The Bendigo Hospital (Bendigo): Jason Fletcher, Julie Smith; Coffs Harbour Health Campus (Coffs Harbour): Raman Azad, Nitin Chavan; Concord Hospital (Concord): Helen Wong; Mark Kol; Royal Darwin Hospital (Darwin): Lewis Campbell; Royal Brisbane and Women's Hospital (Herston, Brisbane): Despoina Koulenti, Therese Starr; Sir Charles Gairdner Hospital (Nedlands): Brigit Roberts, Bradley Wibrow; Redcliffe Hospital (Redcliffe): Timothy Warhurst; St Vincent's Hospital (Toowommba): Meher Chinthamuneedi, Bernal Buitrago Ferney; BELGIUM: Cliniques du Sud Luxembourg (CSL)-Hôpital Saint-Joseph (Arlon): Marc Simon; Chirec Hospital (Braine-l'Alleud): Daniel De Backer; Cliniques Universitaires St Luc (Brussels): Xavier Wittebole; Brugmann University Hospital (Brussels): David De Bels, Cliniques de I'Europe - St-Michel (Brussels): Vincent Collin; University Hospital Antwerp (Edegem): Karolien Dams, Philippe Jorens; Ghent University Hospital (Ghent): Jan De Waele; Jessa Ziekenhuis (Hasselt): Jasperina Dubois; University Hospitals Leuven (Leuven): Jan Gunst; CHU Ambroise Paré (Mons): Lionel Haentjens; Clinique Saint-Pierre (Ottignies): Nicolas De Schryver, Thierry Dugernier; CANADA: St. Michael's Hospital (Toronto): Joao Rezende-Neto, Sandro Rizoli; CHILE: Hospital Clinico Viña del Mar (Viña del Mar): Paul Santillan; CHINA: Jiangsu Province Hospital (Nanjing): Yi Han; Yangpu Hospital of Tongji University (Shanghai): Ewelina Biskup, Changjing Qu; Urumqi General Hospital (Urumqi): Xinyu Li, Wannan Medical College First Affiliated Hospital, Yijishan Hospital (Wuhu): Tao Yu, Lu Weihua; COLOMBIA: Clinica Universitaria Colombia (Bogota): Daniel Molano-Franco, José Rojas, Mederi Hospital (Bogota): Juan Mauricio Pardo Oviedo; Dario Pinilla; Hospital Universitario Fundación Santa Fe (Bogota): Yenny Cardenas, Edgar Celis; Clinica Santa Gracia (Popayan): Mario Arias; CROATIA: Opća bolnica Dubrovnik (Dubrovnik): Anita Vukovic, Maja Vudrag; General Hospital Karlovac (Karlovac): Matija Belavic, Josip Zunic; Clinical Hospital Center Rijeka (Rijeka): Janja Kuharic, Irena Bozanic Kricka; University Hospital Center of Zagreb (Zagreb): Ina Filipovic-Grcic, Boris 
Tomasevic; University Hospital Center Sestre Milosrdnice (Zagreb): Melanija Obraz, Bruna Bodulica; CZECH REPUBLIC: Nemocnice Břeclav (Břeclav): Martin Dohnal; University Hospital Brno (Brno): Jan Malaska, Milan Kratochvil; Municipal Hospital (Havirov): Igor Satinsky, Peter Schwarz; Hospital Karlovy Vary (Karlovy Vary): Zdenek Kos; University Hospital Olomouc (Olomouc): Ladislav Blahut; University Hospital of Ostrava (Ostrava): Jan Maca; Institute for Clinical and Experimental Medicine (Prague): Marek Protus, Eva Kieslichová; DENMARK: Odense University Hospital (Odense): Louise Gramstrup Nielsen, Birgitte Marianne Krogh; ECUADOR: San Vicente de Paúl Hospital (Ibarra): Francisco Rivadeneira; Hospital Oncologico "Dr. Julio Villacreses Colmont" SOLCA (Portoviejo): Freddy Morales, José Mora; Hospital General Puyo (Puyo): Alexandra Saraguro Orozco; Hospital de Especialidades "Eugenio Espejo" (Quito): Diego Rolando MorochoTutillo, Nelson Remache Vargas; Clinica La Merced (Quito): Estuardo Salgado Yepez; Hospital Militar (Quito): Boris Villamagua; EGYPT: Kasr El AINI Hospital, Cairo University (Cairo): Adel Alsisi, Abdelraouf Fahmy; FRANCE: CHU Amiens (Amiens): Hervé Dupont; CHU Angers (Angers): Sigismond Lasocki; Hôpital Beaujon (Clichy): Catherine Paugam-Burtz, Arnaud Foucrier; Centre Hospitalier Compiegne Noyon (Compiègne): Alexandru Nica, Geneviève Barjon; Centre Hospitalier de Lens (Lens): Jihad Mallat; Hôpital Edouard Herriot (Lyon): Guillaume Marcotte; Hôpital Nord (Marseille): Marc Leone, Gary Duclos; Clinique du Millénaire (Montpellier): Philippe Burtin; CHUBichat Claude Bernard (Paris): Philippe Montravers, Enora Atchade; Groupe Hospitalier Paris Saint-Joseph (Paris): Yazine Mahjoub, Benoît Misset; Hôpital Bichat (Paris): Jean-François Timsit, Claire Dupuis; $\mathrm{CHU}$ de Rouen, Hôpital Charles Nicolle (Rouen): Benoît Veber; Centre Hospitalier Yves le Foll (Saint-Brieuc): Matthieu Debarre; Hôpitaux Universitaires de Strasbourg, NHC -Nouvel Hôpital Civil (Strasbourg): Oliver Collange; Hôpitaux Universitaires de Strasbourg, Hôpital de Hautepierre (Strasbourg): Julien Pottecher, Stephane Hecketsweiler; Hôpital Cochin (Paris): Mélanie Fromentin, Antoine Tesnière; GERMANY: University Hospital Giessen (Giessen): Christian Koch, Michael Sander; Universitätsklinikum Schleswig-Holstein (Kiel): Matthias Kott, Gunnar Elke; University Hospital of Leipzig (Leipzig): Hermann Wrigge, Philipp Simon; GREECE: General Hospital of Agios Nikolaos (Agios Nikolados): Anthoula Chalkiadaki, Charalampos Tzanidakis; Democritus University of Thrace (Alexandroupolis): Ioannis Pneumatikos, Eleni Sertaridou: Evangelismos Hospital (Athens): Zafiria Mastora, loannis Pantazopoulos; Hippocrateion General Hospital of Athens (Athens): Metaxia Papanikolaou, Theonymfi Papavasilopoulou; General Hospital Laiko (Athens): John Floros, Virginia Kolonia; University Hospital Attikon (Athens): George Dimopoulos, Chryssa Diakaki; General Hospital Asklepieio Voulas (Athens): Michael Rallis, Alexandra Paridou; General Hospital G. Gennimatas (Athens): Alexandros Kalogeromitros, Vasiliki Romanou; Konstantopouleio Hospital (Athens): Charikleia Nikolaou, Katerina Kounougeri; Agioi Anargiroi General Oncological Hospital of Kifissia (Athens): Evdoxia Tsigou, Vasiliki Psallida; Red Cross Hospital (Athens): Niki Karampela, Konstantinos Mandragos; General Hospital St George (Chania): Eftychia Kontoudaki, Alexandra Pentheroudaki; Thriassio General Hospital of Eleusis (Eleusis): Christos Farazi-Chongouki, Agathi Karakosta; Giannitsa General Hospital (Giannitsa): Isaac Chouris, Vasiliki Radu; University Hospital Heraklion (Heraklion): Polychronis Malliotakis, Sofia Kokkini; Venizelio General Hospital of Heraklion (Heraklion): Eliana Charalambous, Aikaterini Kyritsi; University Hospital of Ioannina (loannina): Vasilios Koulouras, Georgios Papathanakos; General Hospital Kavala (Kavala): Eva Nagky, Clairi Lampiri; Lamia General Hospital (Lamia): Fotios Tsimpoukas, loannis Sarakatsanos; Agios Andrea's General Hospital of Patras (Patras): Panagiotis Georgakopoulos, Ifigeneia Ravani; Tzaneio General Hospital (Pireaus): Athanasios Prekates, Konstantinos Sakellaridis; General Hospital of Pyrgos (Pyrgos Hleias): Christos Christopoulos, Efstratia Vrettou; General Hospotal of Rethymnon (Rethymnon): Konstantinos Stokkos, Anastasia Pentari; Papageorgiou Hospital (Thessaloniki): Kostoula Arvaniti, Kyriaki Marmanidou; Hippokration Hospial (Thessaloniki): Christina Kydona, Georgios Tsoumaropoulos; G. Papanikolaou General Hospital (Thessaloniki): Militisa Bitzani, Paschalina Kontou; Agios Pavlos Hospital (Thessaloniki): Antonios Voudouris, Elli-Nikki, Flioni; General Hospital of Thessaloniki G.Gennimatas (Thessaloniki): Elli Antypa, Eleftheria Chasou; Theagenio Anticancer Hospital (Thessaloniki): Souzana Anisoglou, Eirini Papageorgiou; General Hospital of Trikala (Trikala): Theoniki Paraforou, Agoritsa Tsioka; Achillopoyleio General Hospital Volos (Volos): Antigoni Karathanou; Xanthi General Hospital (Xanthi): Aristeidis Vakalos; INDIA: CIMS Hospital (Ahmedabad): Bhagyesh Shah, Chirag Thakkar; CHL Hospitals (Indore): Nikhilesh Jain; Sanjay Gandhi Postgraduate Institute of Medical Sciences (SGPGIMS) (Lucknow): Mohan Gurjar, Arvind Baronia; Ruby Hall Clinic (Pune): Prachee Sathe, Shilpa Kulkarni; Jubilee Mission Medical College \& Research
Institute (Thrissur): Cherish Paul, John Paul; IRAN: Nemazi Hospital (Shiraz): Mansoor Masjedi; Anesthesiology and Critical Care Research Center, Shiraz University of Medical Sciences (Shiraz): Reza Nikandish, Farid Zand; Shiraz Trauma Hospital (Shiraz): Golnar Sabetian; Shohada Hospital (Tabriz): Ata Mahmoodpoor; Masih Daneshvari Hospital (NRITLD (Tehran): Seyed Mohammadreza Hashemian; ISRAEL: Hadassah Hebrew University Medical Center (Jerusalem): Miklosh Bala; ITALY: Cardarelli Ospedale (Campobasso): Romeo Flocco, Sergio Torrente; PinetaGrande Private Hospital (Castel Volturno): Vincenzo Pota; Arcispedale Sant'Anna (Ferrara): Savino Spadaro, Carlo Volta; University Hospital of Modena (Modena): Massimo Girardis, Giulia Serafini; Ospedale S.Antonio (Padova): Sabrina Boraso, Ivo Tiberio; Azienda Ospedaliera Universitaria Policlinico Paolo Giaccone (Palermo): Andrea Cortegiani, Giovanni Misseri; Azienda Ospedaliero-Universitaria di Parma (Parma): Maria Barbagallo, Davide Nicolotti; Azienda Ospedaliero-Universitaria Pisana (Pisa): Francesco Forfori, Francesco Corradi; Fondazione Policlinico Universitario A.Gemelli IRCCS (Roma): Massimo Antonelli, Gennaro De Pascale; Regina Elena National Cancer Institute of Rome (Roma): Lorella Pelagalli; Azienda Ospedaliero-Universitaria Citta della Salute e della Scienza di Torino, Presidio Ospedaliero Molinette (Torino): Luca Brazzi, Ferdinando Giorgio Vittone; Policlinico Universitario GB Rossi (Verona): Alessandro Russo, Davide Simion; University-Hospital of Foggia (Foggia): Antonella Cotoia, Gilda Cinnella; JAMAICA: University Hospital of the West Indies (Kingston): Patrick Toppin Roxanne Johnson-Jackson; JAPAN: Kameda General Hospital (Kamogawa): Yoshiro Hayashi, Ryohei Yamamoto; Japanese Red Cross Musashino Hospital (Tokyo): Hideto Yasuda, Yuki Kishihara; Okinawa Prectural Chube Hospital (Uruma, Okinawa): Junji Shiotsuka; MEXICO: UMAE Hospital Especialidades Antonio Fraga Mouret-Centro Medico Nacional La Raza IMSS (Mexico City): Luis Alejandro Sanchez-Hurtado, Brigitte Tejeda-Huezo; Hospital Juárez de Mexico (Mexico City): Luis Gorordo; Instituto Nacional de Cancerologia (Mexico City): Silvio A. Namendys-Silva, Francisco J. Garcia-Guillen; Hospital general \# 5 IMSS (Nogales, Sonora): Manuel Martinez; Hospital Regional de Alta Especialidad de la Península de Yucatán (Merida, Yacatan): Erick Romero-Meja, Ever Colorado-Dominguez; NETHERLANDS: Deventer Hospital (Deventer): Huub van den Oever, Karel Martijn Kalff; Medisch Spectrum Twente (Enschede): Wytze Vermeijden, Alexander Daniel Cornet; Tjongerschans Hospital (Heerenveen): Oliver Beck, Nedim Cimic; Zuyderland Medisch Centrum (Heerlen): Tom Dormans, Laura Bormans; Erasmus MC University Medical Center (Rotterdam): Jan Bakker, Ditty Van Duijn; Elisabeth-TweeSteden Ziekenhuis (Tilburg): Gerrit Bosman, Piet Vos; University Medical Center (Utrecht): Dylan de Lange, Jozef Kesecioglu; Diakonessenhuis (Utrecht): Lenneke Haas; OMAN: Khoula Hospital (Muscat): Akram Henein; PARAGUAY: Hospital Regional de Luque (Luque): Ariel M Miranda; PERU: Clínica Ricardo Palma (Lima): Luis Daniel Umezawa Makikado, Gonzalo Ernesto Gianella Malca; Victor Lazarte Echegaray Hospital (Trujillo): Abel Arroyo-Sanchez; POLAND Silesian Hospital Cieszyn (Cieszyn): Agnieszka Misiewska-Kaczur; Wojewodzki Szpital Zesoloby w Koninie (Konin): Frisch Akinyi; First Public Teaching Hospital (Lublin): Miroslaw Czuczwar; Szpital Wojewodzki w Opolu SPZOZ (Opole): Karolina Luczak; SPZZOZ w Ostrowi Mazowieckiej (Ostrow Mazowiecka): Wiktor Sulkowski; Poznan University of Medical Sciences, Regional Hospital in Poznan (Poznan): Barbara Tamowicz, Adam Mikstacki; Centrum Medyczne (Poznan): Beata Swit, Bronisław Baranowski; University Hospital (Poznan): Piotr Smuszkiewicz, Iwona Trojanowska; WSM im. J. Strusia (Poznan): Stanislaw Rzymski; Niepubliczny Zakład Opieki Zdrowotnej Szpital w Puszczykowie im. prof. Stefana Tytusa Dąbrowskiego (Puszczykowo): Mariusz Sawinski, Marta Trosiak; Infant Jesus Teaching Hospital of Warsaw Medical University (Warsaw): Malgorzata Mikaszewska-Sokolewicz; PORTUGAL: Hospital de Braga (Braga): Ricardo Alves, Dina Leal; Centro Hospitalar Algarve (Faro): Andriy Krystopchuk, Pedro Muguel Hilario Mendonca; Centro Hospitalar Universitário Lisboa Central - Hospital Curry Cabral (Lisboa): Rui Antunes Pereira; Centro Hospitalar Universitário Lisboa Norte - Hospital de Santa Maria (Lisboa): Maria Raquel Lopes Marques de Carvalho, Carlos Candeias; Hospital Pedro Hispano (Matosinhos): Elena Molinos, Amélia Ferreira; Centro Hospitalar Sao Joao - Serviço Medicina Intensiva - UCIPU (Porto): Guiomar Castro, José-Artur Paiva; Centro Hospitalar Sao Joao - Serviço Medicina Intensiva - UCIPG (Porto):JoséManuel Pereira; Centro Hospitalar Sao Joao - Infectious Diseases ICU (Porto): Lurdes Santos, Alcina Ferreira; Hospital do Litoral Alentejano (Santiago do Cacém): Dulce Pascoalinho; São Bernardo - Centro Hospitalar Setubal (Setubal): Rosa Ribeiro, Guilherme Domingos; Hospital Vila Franca de Xira (Vila Franca de Xira): Pedro Gomes, David Nora; Centro Hospitalar de Trás-osMontes e Alto Douro (Vila Real): Rui Pedro Costa, Anabela Santos; QATAR: Hamad Medical Corporation (Doha): Ahmed Subhy Alsheikhly; ROMANIA 
Fundeni Clinical Institute (Bucharest): Dana Tomescu, Mihai Popescu; Regional Institute of Oncology (lasi): loana Grigoras, Emilia Patrascanu; RUSSIAN FEDERATION: Krasnodar Regional Hospital \#2 (Krasnodar): Igor Zabolotskikh, Tatiana Musaeva; Krasnoyarsk State Medical University, Krasnoyarsk Regional Clinical Hospital (Krasnoyarsk): Alexey Gritsan, Denis Gaigolnik; Vishnevsky Institute of Surgery (Moscow): Vladimir Kulabukhov; Privolzhskiy District Medical Center (Nizhniy Novgorod): Vladislav Belskiy; Clinical Hospital \# 4 (Perm): NadezhdaZubareva, Maxim Tribulev; SAUDI ARABIA: International Medical Center (Jeddah): Ahmed Abdelsalam, Ayman Aldarsani; King Faisal Specialist Hospital \& Research Centre (Riyadh): Muhammad Al-Khalid; PSMMC (Riyadh): Ghaleb Almekhlafi, Yasser Mandourah; SERBIA: Clinical Centre of Serbia (Belgrade): Bojan Jovanovic, Krstina Doklestic; Clinic for Digestive Surgery (Belgrade): Jelena Velickovic, Dejan Velickovic; Clinical Center Nis, (Nis): Radmilo Jankovic, Anita Vukovic; Oncology Institute of Vojvodina (Sremska Kamenica): Svetlana Skoric-Jokic, Dragana Radovanovic; SOUTH AFRICA: Charlotte Maxeke Johannesburg Academic Hospital (Johannesburg): Guy Richards, Ahmad Alli; SPAIN: Complejo Hospitalario Universitario de Albacete (Albacete): Maria del Carmen Cordoba Nielfa, Rafael Sánchez Iniesta; Parc de Salut Mar (Barcelona): Adela Benítez-Cano Martínez, Carlos Garcia Bernedo; Hospital Delfos (Barcelona): Santiago Alberto Picos Gil; Vall d'Hebron University Hospital (Barcelona): Xavier Nuvials, Jordi Rello; Hospital Universitario de Basurto (Bilbao): Joseba Gonzalez Garcia, Jose Manuel Garcia Peña; Hospital General Universitario Santa Lucia (Cartagena): Roberto Jimenez, Luis Herrera; Hospital General Universitari de Castelló (Castelló): Laura Galarza Barrachina, Ignacio Catalan Monzon; Hospital General Universitario de Ciudad Real (Ciudad Real): Francisco Javier Redondo, Ruben Villazala; Hospital Costa de la Luz (Huelva): Diego Fernando Matallana Zapata, Isabel Maria Villa Lopez; Hospital Universitari de Bellvitge (L'Hospitalet de Llobregat): Gabriel Moreno-Gonzalez, Juan Carlos Lopez-Delgado; Hospital Universitario de Canarias (La Laguna): Jorge Solera Marin; Hospital Universitario Severo Ochoa (Léganes): Purificacion Sanchez-Zamora; Hospital Universitari Arnau de Vilanova (Lleida): Montserrat Vallverdú Vidal; Hospital Quirón Campo de Gibraltar (Cádiz): Jesús Flores González; Hospital Universitarion del Henares (Madrid): Irene Salinas, Cecilia Hermosa; Hospital Universitario La Paz (Madrid): Emilio Maseda; Hospital Clinico San Carlos (Madrid): Fernando MartinezSagasti, Sara Domingo-Marín; Central de la Defensa Gomez Ulla (Madrid): Johanna Abril Victorino; Hospital 12 de Octubre (Madrid): Raquel Garcia-Alvarez, Pablo López-Arcas Calleja; Hospital Universitario de Malaga (Malaga): Maria-Victoria de la Torre-Prados; CHU Ourense (Ourense): Pablo Vidal-Cortes, Lorena del Río-Carbajo; Complejo Hospitalario de Navarra (Pamplona): Javier Izura, Victoria Minguez; Hospital Universitari Mutua Terrassa (Terrassa): Josep Trenado Alvarez, Anna Parera Prous; Complejo Hospitalario de Toledo (Toledo): Daniel Paz; Hospital Verge de la Cinta (Tortosa): Ferran Roche-Campo; Hospital Clínico Universitario de Valencia (Valencia): Gerardo Aguilar, Javier Belda; Rio Hortega University Hospital (Valladolid): Jesus Rico-Feijoo, Cesat Aldecoa; Hospital Clinico Universitario Lozano Blesa (Zaragoza): Begoña Zalba-Etayo; SWITZERLAND: Kantonsspital Frauenfeld (Frauenfeld): Martin Lang; Alexander Dullenkopf; THAILAND: Faculty of Medicine Vajira Hospital, Navamindradhiraj University (Bangkok): Konlawij Trongtrakul; Anusang Chtsomkasem; TURKEY: Düzce University Hospital (Duzce): Türkay Akbaş; Ankara University School of Medicine (Ankara): Mustafa Necmettin Unal, Menekse Ozcelik; Akdeniz University Medical School (Antalya): Ayca Gumus, Atilla Ramazanoglu; Trakya University Medical Faculty (Edirne): Dilek Memis, Inal Mehmet; Istanbul University-Cerrahpasa, Cerrahpasa Medical School (Istanbul): Yalim Dikmen, Seval Urkmez; Haydarpaşa Numune Training and Research Hospital (Istanbul): Asu Ozgultekin; Istanbul University Cerrahpasa Medical School Hospital (Istanbul): Oktay Demirkiran; Medipol Mega Hospitals Complex (Istanbul): Nesrin Ahu Aslan, Deniz Kizilaslan; Uludag University, School of Medicine (Nilüfer/Bursa): Ferda Kahveci, Nurdan Ünlü; Elazig Training \& Research Hospital (Elazig): Zeynep Ozkan; UNITED KINGDOM: Aberdeen Royal Infirmary (Aberdeen): Callum Kaye, Jan Jansen; Antrim Area Hospital (Antrim): Orla O'Neill, Christopher Nutt; Barnet General Hospital, RFL NHS FT (Barnet): Rajeev Jha, Nicolas Hooker; Basingsoke \& North Hampshire Hospital (Basingstoke): Irina Grecu, Christina Petridou; Royal Victoria Hospital (Belfast): Murali Shyamsundar, Lia McNamee; Ulster Hospital (Belfast): John Trinder, Samantha Hagan; Belfast City Hospital (Belfast): Catriona Kelly, Jonathon Silversides; Brighton and Sussex University Hospitals (Brighton): Casiano Barrera Groba, Owen Boyd; West Suffolk Hospital NHS Foundation Trust (Bury St Edmunds): Kaushik Bhowmick, Sally Humphreys; Cambridge University Hospitals NHS Foundation Trust and University of Cambridge (Cambridge): Charlotte Summers, Petra Polgarova; Western Sussex NHS
Foundation Trust, St Richard's Hospital (Chichester, West Sussex): Michael Margarson, Justin Dickens; Colchester General Hospital (Colchester): Suzanne Pearson, Elaine Chinery; Altnagelvin Hospital (Derry): Noel Hemmings, Sinead O'Kane; Ninewells Hospital (Dundee): Pauline Austin, Stephen Cole; Medway NHS Foundation Trust (Gillingham): Catherine Plowright, Roberta Box; Queen Elizabeth University Hospital (Glasgow): Christopher Wright, Lorna Young; Royal Surrey County Hospital (Guildford): Ben Creagh-Brown, Laura Montague; Aintree University Hospital (Liverpool): Robert Parker; Ben Morton; Guy's and St Thomas Hospitals (London): Marlies Ostermann, Julia Bilinska; University Hospital Lewisham (London): Bernd Oliver Rose, Rosie Reece-Anthony; St Georges University Hospitals NHS Foundation Trust (London): Christine Ryan, Mark Hamilton; King's College Hospital (London): Philip Hopkins, Julia Wendon; Luton and Dunstable Hospital (Luton): Giovanni Brescia, Nazia ljaz; Maidstone and Tunbridge Wells NHS Trust Hospital (Maidstone): James Wood, Michelle George; Prince Charles Hospital (Merthyr Tydfil): Piroska Toth-Tarsoly; Northumbria Specialist Emergency Care Hospital (Newcastle Upon Tyne): Bryan Yates, Maureen Armstrong; Royal Victoria Infirmary (Newcastle Upon Tyne): Carmen Scott, Christine Boyd; Royal Gwent Hospital (Newport): Tamas Szakmany, David Rees; Kings Mill Hospital (Nottingham): Paul Pulak, Mandy Coggon; Royal Oldham Hospital (Oldham): Bhaskar Saha, Linda Kent; Royal Glamorgan Hospital (Pontyclun): Bethan Gibson; Poole Hospital NHS FT (Poole): Julie Camsooksai, Henrik Reschreiter; East Surrey Hospital (Redhill): Pat Morgan, Sivatharshini Sangaralingham; Conquest Hospital (St Leonards-onsea): Alastair Lowe, Petr Vondras; Lister Hospital (Stevenage): Sunil Jamadarkhana, Carina Cruz; University Hospital of North Tees (Stockton-on-Tees): Rakesh Bhandary; Sunderland Royal Hospital (Sunderland): Peter Hersey, Julie Furneval; Musgrove Park Hospital (Taunton): Richard Innes, Patricia Doble; Warwick Hospital (Warwick): Ben Attwood, Penny Parsons; Watford General Hospital (Watford): Valerie Page, Xiaobei Zhao; Royal Hampshire County Hospital (Winchester): Irina Grecu, Julian Dalton; UNITED ARAB EMIRATES: Sheikh Khalifa Medical City (Abu Dhabi): Mohammed Hegazy, Yasser Awad; UNITED STATES: Cleveland Clinic (Cleveland): Douglas Naylor, Amanda Naylor; Detroit Medical Center (Detroit): Sarah Lee; University of South Alabama Medical Center (Mobile, AL): Sidney Brevard, Noelle Davis.

\section{Funding}

AbSeS is a Trials Group Study of the European Society of Intensive Care Medicine. The study was supported by a Pfizer investigator-initiated research grant.

\section{Compliance with ethical standards}

\section{Conflicts of interest}

Received grants related to the submitted work: S. Blot (Pfizer). Received honoraria or grants outside the submitted work: M. Antonelli (Fresenius, Pfizer, Toray); J. De Waele (Research Foundation Flanders, Pfizer, Bayer, MSD); C. Eckmann (Merck, Pfizer); J. Lipman (MSD, Pfizer); E. Maseda (Astellas Pharma, Pfizer, MSD); All other authors: no conflict of interest.

\section{Open Access}

This article is distributed under the terms of the Creative Commons Attribution-NonCommercial 4.0 International License (http://creativecommons.org/ licenses/by-nc/4.0/), which permits any noncommercial use, distribution, and reproduction in any medium, provided you give appropriate credit to the original author(s) and the source, provide a link to the Creative Commons license, and indicate if changes were made.

\section{Publisher's Note}

Springer Nature remains neutral with regard to jurisdictional claims in published maps and institutional affiliations.

Received: 28 June 2019 Accepted: 9 October 2019 Published online: 29 October 2019

\section{References}

1. Vincent JL, Rello J, Marshall J et al (2009) International study of the prevalence and outcomes of infection in intensive care units. JAMA 302:2323-2329. https://doi.org/10.1001/jama.2009.1754 
2. Kübler A, Adamik B, Ciszewicz-Adamiczka B, Ostrowska E (2015) Severe sepsis in intensive care units in Poland - a point prevalence study in 2012 and 2013. Anaesthesiol Intensive Ther 47:315-319. https://doi. org/10.5603/AIT.2015.0047

3. Sakr Y, Jaschinski U, Wittebole $X$ et al (2018) Sepsis in intensive care unit patients: worldwide data from the intensive care over nations audit. Open Forum Infect Dis 5:ofy313. https://doi.org/10.1093/ofid/ofy313

4. Blot S, De Waele JJ (2005) Critical issues in the clinical management of complicated intra-abdominal infections. Drugs 65:1611-1620

5. Montravers P, Blot S, Dimopoulos G et al (2016) Therapeutic management of peritonitis: a comprehensive guide for intensivists. Intensive Care Med 42:1234-1247. https://doi.org/10.1007/s00134-016-4307-6

6. Solomkin JS, Mazuski JE, Bradley JS et al (2010) Diagnosis and management of complicated intra-abdominal infection in adults and children: guidelines by the Surgical Infection Society and the Infectious Diseases Society of America. Surg Infect (Larchmt) 11:79-109. https://doi. org/10.1089/sur.2009.9930

7. Azuhata T, Kinoshita K, Kawano D et al (2014) Time from admission to initiation of surgery for source control is a critical determinant of survival in patients with gastrointestinal perforation with associated septic shock. Crit Care 18:R87. https://doi.org/10.1186/cc13854

8. Sartelli M, Chichom-Mefire A, Labricciosa FM et al (2017) The management of intra-abdominal infections from a global perspective: 2017 WSES guidelines for management of intra-abdominal infections. World J Emerg Surg 12:29. https://doi.org/10.1186/s13017-017-0141-6

9. Tolonen M, Coccolini F, Ansaloni L et al (2018) Getting the invite list right: a discussion of sepsis severity scoring systems in severe complicated intra-abdominal sepsis and randomized trial inclusion criteria. World J Emerg Surg 13:17. https://doi.org/10.1186/s13017-018-0177-2

10. Blot S, De Waele JJ, Vogelaers D (2012) Essentials for selecting antimicrobial therapy for intra-abdominal infections. Drugs 72:e17-e32. https://doi. org/10.2165/11599800-0000000000-00000

11. Calandra T, Cohen J (2005) International Sepsis Forum Definition of Infection in the ICU Consensus Conference The international sepsis forum consensus conference on definitions of infection in the intensive care unit. Crit Care Med 33:1538-1548. https://doi.org/10.1097/01.ccm.0000 68253.91200 .83

12. Trouillet JL, Chastre J, Vuagnat A et al (1998) Ventilator-associated pneumonia caused by potentially drug-resistant bacteria. Am J Respir Crit Care Med 157:531-539. https://doi.org/10.1164/ajrccm.157.2.9705064

13. Singer M, Deutschman CS, Seymour CW, et al (2016) The third international consensus definitions for sepsis and septic shock (Sepsis-3). JAMA 315(8):801-810. https://doi.org/10.1001/jama.2016.0287

14. The European Committee on Antimicrobial Susceptibility Testing. Breakpoint tables for interpretation of MICs and zone diameters. Version 5.0 2015. http://www.eucast.org. Accessed 31 Mar 2014

15. Kadri SS, Adjemian J, Lai YL et al (2018) Difficult-to-treat resistance in gram-negative bacteremia at 173 US hospitals: retrospective cohort analysis of prevalence, predictors, and outcome of resistance to all firstline agents. Clin Infect Dis 67:1803-1814. https://doi.org/10.1093/cid/ ciy378

16. Kadri SS, Lai YLE, Ricotta EE et al (2019) External validation of difficultto-treat resistance prevalence and mortality risk in gram-negative bloodstream infection using electronic health record data from 140 US hospitals. Open Forum Infect Dis 6:ofz110. https://doi.org/10.1093/ofid/ ofz110

17. Versporten A, Zarb P, Caniaux I et al (2018) Antimicrobial consumption and resistance in adult hospital inpatients in 53 countries: results of an internet-based global point prevalence survey. Lancet Glob Health 6:e619-e629. https://doi.org/10.1016/S2214-109X(18)30186-4

18. Vogelaers D, De Bels D, Forêt F et al (2010) Patterns of antimicrobial therapy in severe nosocomial infections: empiric choices, proportion of appropriate therapy, and adaptation rates - a multicentre, observational survey in critically ill patients. Int J Antimicrob Agents 35:375-381. https ://doi.org/10.1016/j.ijantimicag.2009.11.015

19. Solomkin JS, Yellin AE, Rotstein OD et al (2003) Ertapenem versus piperacillin/tazobactam in the treatment of complicated intraabdominal infections: results of a double-blind, randomized comparative phase III trial. Ann Surg 237:235-245. https://doi.org/10.1097/01.SLA.0000048551 .32606 .73

20. Solomkin JS, Wilson SE, Christou NV et al (2001) Results of a clinical trial of clinafloxacin versus imipenem/cilastatin for intraabdominal infections. Ann Surg 233:79-87. https://doi.org/10.1097/00000658-20010 1000-00013

21. Solomkin JS, Reinhart HH, Dellinger EP et al (1996) Results of a randomized trial comparing sequential intravenous/oral treatment with ciprofloxacin plus metronidazole to imipenem/cilastatin for intra-abdominal infections. The Intra-Abdominal Infection Study Group. Ann Surg 223:303-315. https://doi.org/10.1097/00000658-199603000-00012

22. de Ruiter J, Weel J, Manusama E et al (2009) The epidemiology of intra-abdominal flora in critically ill patients with secondary and tertiary abdominal sepsis. Infection 37:522-527. https://doi.org/10.1007/s1501 0-009-8249-6

23. Blot K, Hammami N, Blot S et al (2019) Increasing burden of Escherichia coli, Klebsiella pneumoniae, and Enterococcus faecium in hospital-acquired bloodstream infections (2000-2014): a national dynamic cohort study. Infect Control Hosp Epidemiol 40:705-709. https://doi.org/10.1017/ ice.2019.59

24. Martínez ML, Ferrer R, Torrents E et al (2017) Impact of source control in patients with severe sepsis and septic shock. Crit Care Med 45:11-19. https://doi.org/10.1097/CCM.0000000000002011

25. Montravers P, Dupont H, Gauzit R et al (2006) Candida as a risk factor for mortality in peritonitis. Crit Care Med 34:646-652. https://doi. org/10.1097/01.CCM.0000201889.39443.D2

26. Knitsch W, Vincent JL, Utzolino S et al (2015) A randomized, placebocontrolled trial of preemptive antifungal therapy for the prevention of invasive candidiasis following gastrointestinal surgery for intra-abdominal infections. Clin Infect Dis 61:1671-1678. https://doi.org/10.1093/cid/civ707

27. van de Groep K, Verhoeff TL, Verboom DM et al (2019) Epidemiology and outcomes of source control procedures in critically ill patients with intraabdominal infection. J Crit Care 52:258-264. https://doi.org/10.1016/j. jcrc.2019.02.029 